Research Article

\title{
Computing Analysis of Zagreb Indices for Generalized Sum Graphs under Strong Product
}

\author{
Muhammad Javaid (D), ${ }^{1}$ Saira Javed $\left(\mathbb{D},{ }^{1}\right.$ Abdulaziz Mohammed Alanazi, ${ }^{2}$ \\ and Majdah R. Alotaibi ${ }^{3}$ \\ ${ }^{1}$ Department of Mathematics, School of Science, University of Management and Technology, Lahore 54770, Pakistan \\ ${ }^{2}$ Department of Mathematics, University of Tabuk, Tabuk 71412, Saudi Arabia \\ ${ }^{3}$ Department of Chemistry, University of Tabuk, Tabuk 71412, Saudi Arabia
}

Correspondence should be addressed to Muhammad Javaid; javaidmath@gmail.com

Received 29 November 2020; Revised 13 December 2020; Accepted 20 December 2020; Published 9 January 2021

Academic Editor: Muhammad Faisal Nadeem

Copyright (c) 2021 Muhammad Javaid et al. This is an open access article distributed under the Creative Commons Attribution License, which permits unrestricted use, distribution, and reproduction in any medium, provided the original work is properly cited.

\begin{abstract}
Numerous studies based on mathematical models and tools indicate that there is a strong inherent relationship between the chemical properties of the chemical compounds and drugs with their molecular structures. In the last two decades, the graphtheoretic techniques are frequently used to analyse the various physicochemical and structural properties of the molecular graphs which play a vital role in chemical engineering and pharmaceutical industry. In this paper, we compute Zagreb indices of the generalized sum graphs in the form of the different indices of their factor graphs, where generalized sum graphs are obtained under the operations of subdivision and strong product of graphs. Moreover, the obtained results are illustrated with the help of particular classes of graphs and analysed to find the efficient subclass with dominant indices.
\end{abstract}

\section{Introduction}

In many fields (chemistry, physics, computer science, and electrical networks) various physicochemical and structural properties such as melting point, boiling point, chemical bonds, bond energy, solubility, surface tension, critical temperature, connectivity, stability, density, and polarizability are studied with the help of various TIs (degree-based, distance-based, and polynomial-based). Moreover, degreebased TIs have been used as a powerful approach to discover many new drugs, such as anineoplastics, anticonvulsants, antiallergics, antimalarials, and silico generation (see [1]). Therefore, this practice has proven that the TIs and the quantitative structure-activity (or structure-property) relationships (QSAR or QSPR) have presented a foundation stone in chemical engineering and pharmaceutical industry for the process of the drug design and discovery (see $[2,3]$ ).

Let $\Omega$ be a collection of (molecular) graphs in which each graph is considered as a simple graph without multiedges and loops. A topological index (TI) is a function
Top: $\Omega \longrightarrow \mathbb{R}$ that assigns a real number to each element (graph) of $\Omega$, where $\mathbb{R}$ is a set of real numbers. Moreover, for two graphs $G_{1}$ and $G_{2}$, Top $\left(G_{1}\right)=\operatorname{Top}\left(G_{2}\right)$ if and only if $G_{1}$ is isomorphic to $G_{2}$. Mostly, TIs are computed for the hydrogen-suppressed molecular graphs in which the atoms are represented by nodes and bonds between them by edges. In 1947, Wiener index (path number), first distance-based TI, is utilized in the study of paraffin's boiling point [4]. Gutman and Trinajstic [5] calculated total $\pi$-electrons energy of the molecules through a degree-based TI called as the first Zagreb index (FZI). They also studied the various properties of the second Zagreb index (SZI) in the same paper. In chemical graph theory, many more TIs are introduced in [6], but degree-based TIs are prominent than others. For more details, we refer to $[7,8]$.

On the other hand, operations on graphs (addition, complement, deletion, switching, subdivision, union, intersection, and product) also play a very important role in the construction of new graphs and structures. Yan et al. [9] introduced the four operations $S_{1}, R_{1}, Q_{1}$, and $T_{1}$ on the 
subdivision of a graph and obtained the Wiener index of these resultant graphs $\left(S_{1}(G), R_{1}(G), Q_{1}(G), T_{1}(G)\right)$. For $\Phi_{1} \in\left\{S_{1}, R_{1}, Q_{1}, T_{1}\right\}$, Taeri and Eliasi [10] defined the $\Phi_{1}$-sum graphs $\left(G_{1+\Phi_{1}} G_{2}\right)$ using the Cartesian product on graphs $\Phi_{1}\left(G_{1}\right)$ and $G_{2}$, where $G_{1}$ and $G_{2}$ are connected graphs. They also studied the Wiener index of these $\Phi_{1}$-sum graphs. Liu et al. [11] constructed $\Phi$-sum graphs with the help of the Cartesian product on the graphs $\Phi\left(G_{1}\right)$ and $G_{2}$ and calculated the first general Zagreb index for these graphs, i.e., $M_{1}^{\alpha}\left(\left(G_{1+\Phi_{1}} G_{2}\right)\right)$. Liu et al. [12] introduced the generalized $\Phi$-sum ( $\Phi_{k}$-sum) graphs with the help of the Cartesian product on the graphs $\Phi_{k}\left(G_{1}\right)$ and $G_{2}$, where $k$ represents some integral value. Moreover, they calculated the mathematical expressions of the Zagreb indices for these graphs, i.e., $M_{1}\left(\left(G_{1+\Phi_{1}} G_{2}\right)\right)$ and $M_{2}\left(\left(G_{1+\Phi_{k}} G_{2}\right)\right)$. Furthermore, Awais et al. [13,14] computed the forgotten topological and hyper-Zagreb indices of generalized F-sum graphs based on Cartesian product in terms of its factor graphs. Recently, Awais et al. [15] computed the first general Zagreb index of $F_{k}$-sum graphs in terms of TIs of their factor graphs.

In the current study, we study the generalized $\Phi$-sum graphs which are obtained under the operation of strong product on the graphs $\Phi_{k}\left(G_{1}\right)$ and $G_{2}$, where $\Phi_{k} \in\left\{S_{k}, R_{k}, Q_{k}, T_{k}\right\}$ and $k$ is some counting number. Mainly, we compute the Zagreb indices of these generalized $\Phi$-sum graphs based on strong product such as $M_{1}\left(\left(G_{1 \bowtie \Phi_{k}} G_{2}\right)\right)$ and $M_{2}\left(\left(G_{1 \bowtie \Phi_{k}} G_{2}\right)\right)$. Moreover, a comparison is also organized of the generalized $\Phi$-sum graphs $\left(G_{1 \bowtie S_{k}} G_{2}\right), \quad\left(G_{1 \otimes R_{k}} G_{2}\right), \quad\left(G_{1 \otimes Q_{k}} G_{2}\right), \quad$ and $\left(G_{1 \otimes T_{k}} G_{2}\right)$ with respect to both the Zagreb indices $\left(M_{1}\right.$ and $M_{2}$ ). The rest of the paper is settled as follows: Section 2 covers basic notions, Section 3 predicated on main results, and conclusively Section 4 included the application and conclusion."

\section{Preliminaries}

A graph $G_{1}$ is a structure consisting of two finite sets of vertices $V(G)$ and edges $E(G)$ in which pairs of vertices are connected by edges. In particular, a graph will refer to a simple undirected graph if each edge connects two distinct vertices and there are no parallel edges. Throughout the paper, the order of $G_{1}$ is $\left|V\left(G_{1}\right)\right|=n_{G_{1}}$, and the size of a $G_{1}$ is $\left|E\left(G_{1}\right)\right|=e_{G_{1}}$. Given two vertices $p$ and $z$, if $p z \in G_{1}$, then $p$ and $z$ are said to be adjacent. The strength of edges which are incident on any node $p \in V\left(G_{1}\right)$ is known as its degree $d_{G_{1}}(p)$ [16]. Here, we defined few topological indices.

Definition 1. Let $G_{1}$ be a simple undirected graph. The first Zagreb index $\left(M_{1}\left(G_{1}\right)\right.$ and second Zagreb index $\left(M_{2}\left(G_{1}\right)\right)$ are:

$$
\begin{aligned}
& M_{1}\left(G_{1}\right)=\sum_{p \varepsilon V\left(G_{1}\right)}\left[d_{G_{1}}(p)\right]^{2}=\sum_{p z \varepsilon E\left(G_{1}\right)}\left[d_{G_{1}}(p)+d_{G_{1}}(z)\right], \\
& M_{2}\left(G_{1}\right)=\sum_{p z \varepsilon E\left(G_{1}\right)}\left[d_{G_{1}}(p) \times d_{G_{1}}(z)\right] .
\end{aligned}
$$

In 1972, Trinajsti and Gutman [5] introduced these two TIs which are used in study of structure-based properties of (molecular) graphs (see [17-19]). In 1960, Sabidussi [6] introduced the strong product $\left(G_{1} \otimes G_{2}\right)$ for two graphs $G_{1}$ and $G_{2}$ with vertex set as Cartesian product $V\left(G_{1} \otimes G_{2}\right)=$ $V\left(G_{1}\right) \times V\left(G_{2}\right)$ such that $\left(p_{1}, p_{2}\right)$ and $\left(z_{1}, z_{2}\right)$ will be adjacent in $G_{1} \otimes G_{2}$ iff $p_{1}=z_{1}$ and $p_{2}$ is adjacent to $z_{2}$ or $p_{2}=z_{2}$ and $p_{1}$ is adjacent to $z_{1}$ or $p_{1}$ is adjacent to $z_{1}$ and $p_{2}$ is adjacent to $z_{2}$. Strong product is union of tensor product and Cartesian product.

Definition 2. The four generalized operations related to the subdivision of graphs defined in [15] are given as follows:

(i) $k$-subdivision operation $S_{k}=S_{k}\left(G_{1}\right)$ can be made by adding $k$ new node in each $u v \varepsilon E\left(G_{1}\right)$ of $G_{1}$, where $k \geq 1$ is an integral value

(ii) $k$-semitotal-point graph $R_{k}=R_{k}\left(G_{1}\right)$ with node set $V\left(R_{k}\right)=V\left(G_{1}\right) \cup k E\left(G_{1}\right)$ and link set $E\left(R_{k}\right)=$ $E\left(S_{k}\right) \cup E\left(G_{1}\right)$

(iii) $k$-semitotal line graph $Q_{k}=Q_{k}\left(G_{1}\right)$ with node set $V\left(Q_{k}\right)=V\left(G_{1}\right) \cup k E\left(G_{1}\right)$ and link set $E\left(Q_{k}\right)=$ $E\left(S_{k}\right) \cup E\left(L_{k}\right)$.

(iv) $k$-total point graph $T_{k}=T_{k}\left(G_{1}\right)$ with node set $V\left(T_{k}\right)=V\left(G_{1}\right) \cup k E\left(G_{1}\right)$ and link set $E\left(T_{k}\right)=$ $E\left(S_{k}\right) \cup E\left(L_{k}\right) \cup E\left(G_{1}\right)$ (for more details, see Figure 1).

Definition 3. Let $G_{1}$ and $G_{2}$ be two graphs, $\Phi_{k} \in\left\{S_{k}, R_{k}, Q_{k}, T_{k}\right\}$ is an operation, and $\Phi_{k}\left(H_{1}\right)$ is obtained after applying $\Phi_{k}$ on $G_{1}$ having edge-set $E\left(\Phi_{k}\left(G_{1}\right)\right)$ and node set $V\left(\Phi_{k}\left(G_{1}\right)\right)$. The generalized $\Phi$-sum graph $\left(G_{1 \bowtie \Phi_{k}} G_{2}\right)$ is a graph having node set:

$$
\begin{aligned}
V\left(G_{1 \bowtie \Phi_{k}} G_{2}\right) & =V\left(\Phi_{k}\left(G_{1}\right)\right) \times V\left(G_{2}\right) \\
& =\left(V\left(G_{1}\right) \cup k E\left(G_{1}\right)\right) \times V\left(G_{2}\right),
\end{aligned}
$$

such that two nodes $\left(p_{1}, z_{1}\right) \&\left(p_{2}, z_{2}\right)$ of $V\left(G_{1 \bowtie \Phi_{k}} G_{2}\right)$ are adjacent iff $p_{1}=p_{2} \in V\left(G_{1}\right)$ and $\left(z_{1}, z_{2}\right) \in E\left(G_{2}\right)$ or $z_{1}=z_{2} \in V\left(G_{2}\right)$ and $\left(p_{1}, p_{2}\right) \in E\left(\Phi_{k}\left(G_{1}\right)\right) \quad$ or $\left[\left(z_{1}, z_{2}\right) \in E\left(G_{2}\right)\right.$ and $\left.\left(p_{1}, p_{2}\right) \in E\left(\Phi_{k}\left(G_{1}\right)\right)\right]$, where $k \geq 1$ is a positive number. We noticed that the generalized $\Phi$-sum graphs $\left(G_{1 \bowtie \Phi_{k}} G_{2}\right)$ contain $\left|V\left(G_{2}\right)\right|$ copies of graphs $\Phi_{k}\left(G_{1}\right)$ that are labeled with the nodes of $G_{2}$. For more details, see Figures 2 and 3.

\section{Main Results}

Now, we will prove the key results of $M_{1}\left(G_{1} \otimes_{\Phi_{k}} G_{2}\right)$ and $M_{2}\left(G_{1} \otimes_{\Phi_{k}} G_{2}\right)$ in terms of its factor graphs $G_{1}$ and $G_{2}$. We assume that $G_{1}$ and $G_{2}$ be two simple, undirected, and connected graphs with order and size, $\left|V\left(G_{1}\right)\right|=$ $n_{G_{1}} \&\left|E\left(G_{1}\right)\right|=e_{G_{1}} \quad$ and $\quad\left|V\left(G_{2}\right)\right|=n_{G_{2}} \&\left|E\left(G_{2}\right)\right|=e_{G_{2}}$ respectively.

Theorem 1. Let $G_{1}$ and $G_{2}$ be two connected graphs such that $\left|V\left(G_{1}\right)\right| \geq 3,\left|V\left(G_{2}\right)\right| \geq 2$. For $k \geq 1$, 


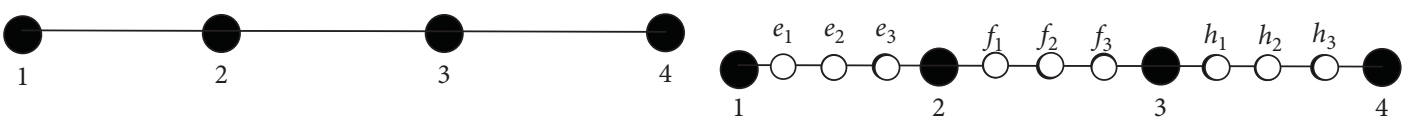

(a)

(b)

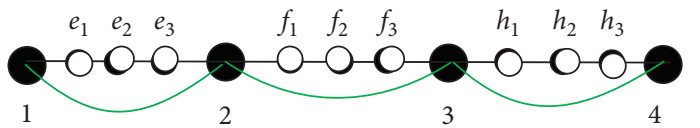

(c)

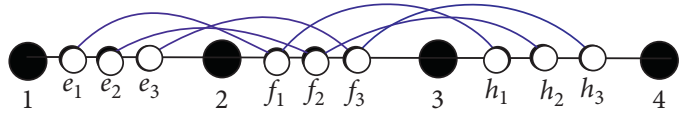

(d)

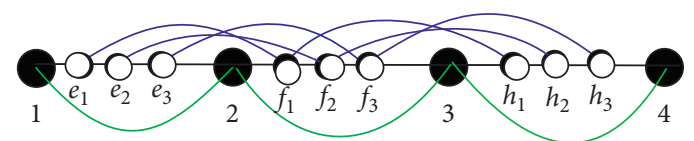

(e)

Figure 1: (a) $G_{1} \cong P_{4}$. (b) $S_{3}\left(G_{1}\right)$. (c) $R_{3}(G p 1)$. (d) $Q_{3}\left(G_{1}\right)$. (e). $T_{3}\left(G_{1}\right)$.

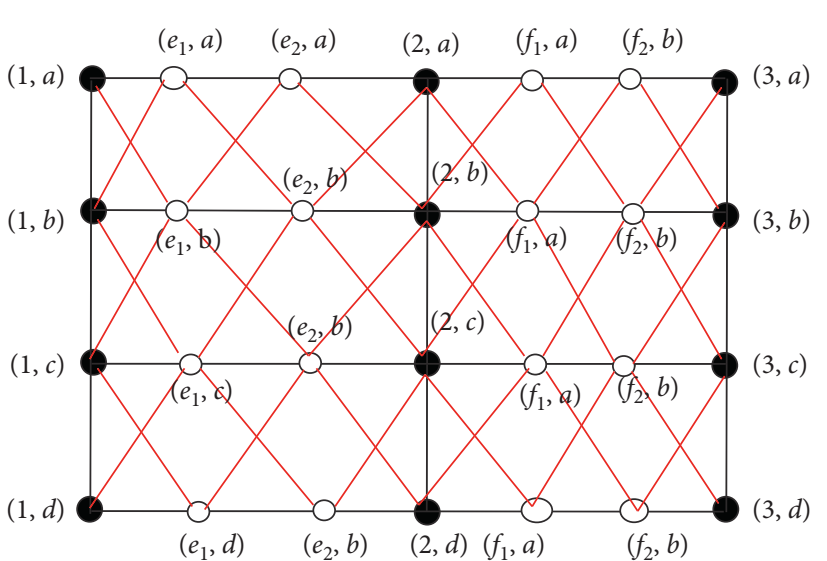

(a)

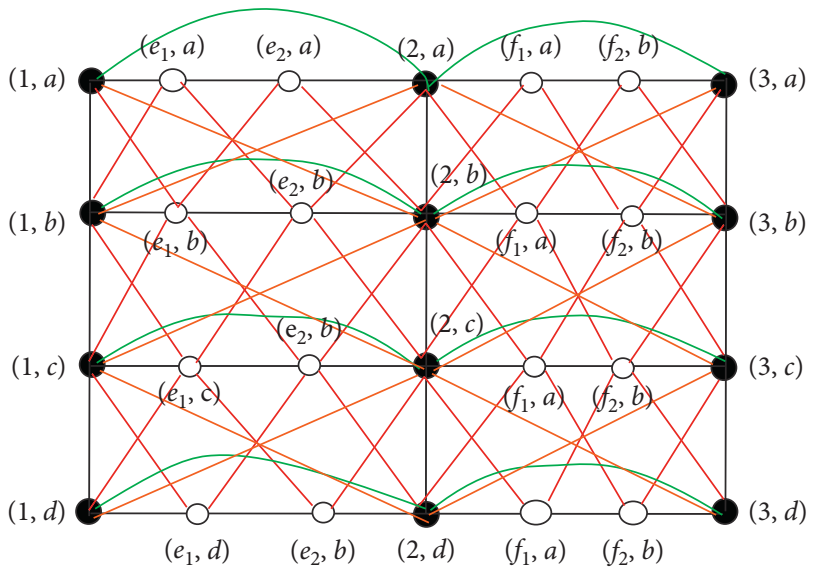

(b)

Figure 2: Right: $=P_{3 \bowtie S_{2}} P_{4}$. Left: $=P_{3 \bowtie R_{2}} P_{4}$.

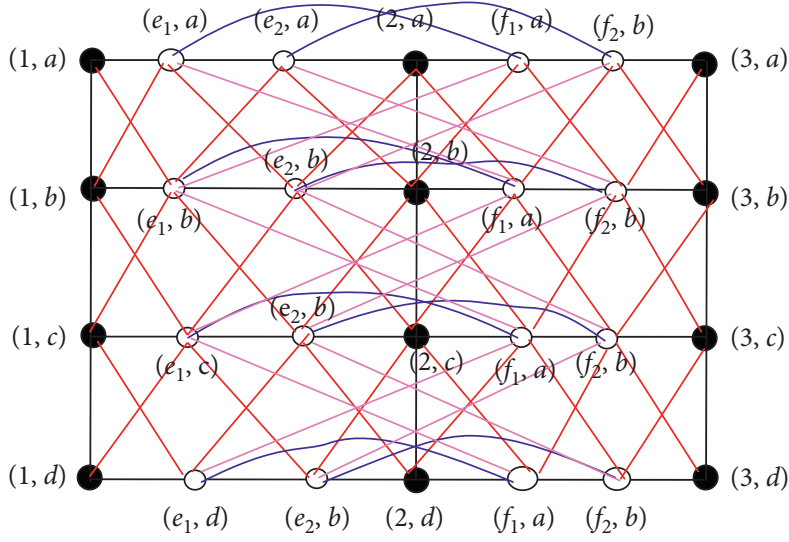

(a)

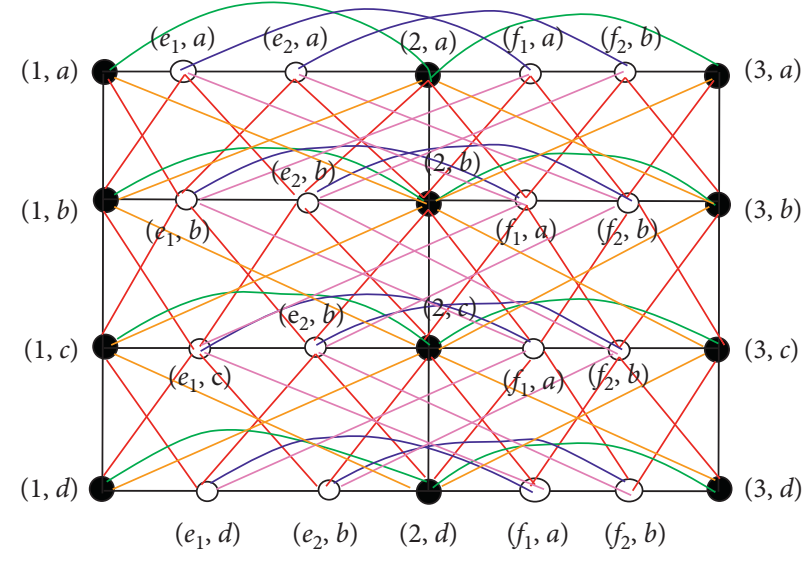

(b)

Figure 3: Right: $=P_{3 \bowtie Q_{2}} P_{4}$. Left: $=P_{3 \bowtie T_{2}} P_{4}$. 


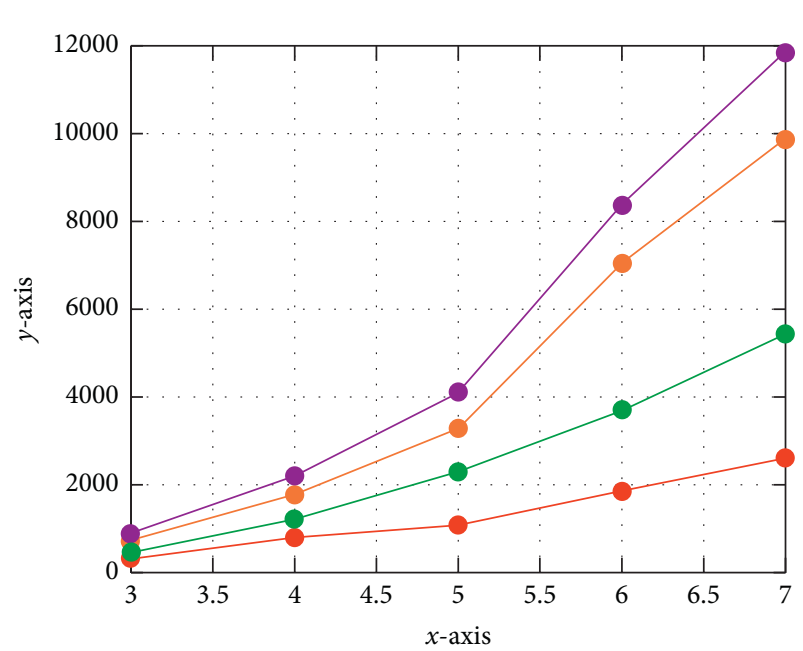

(a)

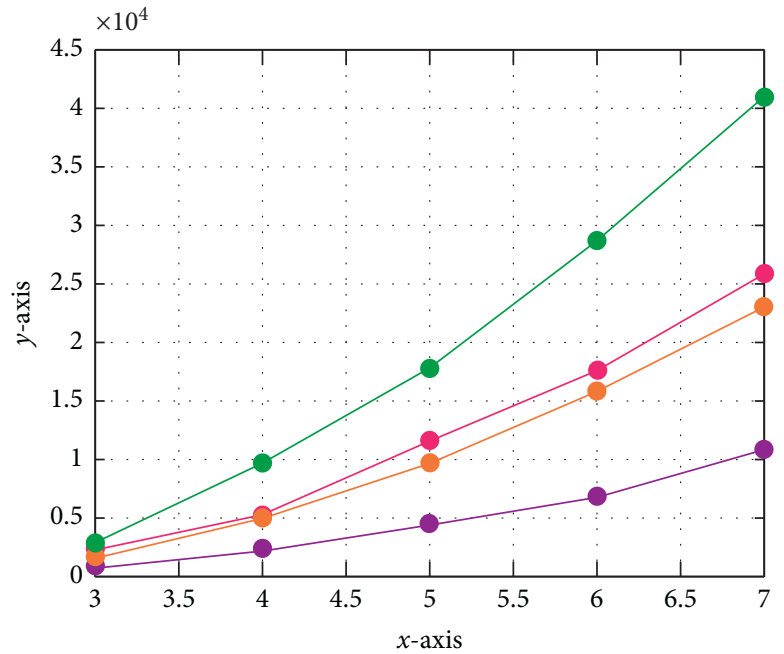

(b)

FiguRe 4: (a) Graphical representation of $M_{1}\left(P_{n_{S 1}} \otimes P_{m}\right), M_{1}\left(P_{n_{\mathrm{Ol}}} \otimes P_{m}\right), M_{1}\left(P_{n_{R 1}} \otimes P_{m}\right)$, and $M_{1}\left(P_{n_{T 1}} \otimes P_{m}\right)$ by red, green, orange, and purple colour, respectively. (b) $M_{2}\left(P_{n_{S 1}} \otimes P_{m}\right), M_{2}\left(P_{n_{\mathrm{Q} 1}} \otimes P_{m}\right), M_{2}\left(P_{n_{R 1}}^{\mathrm{Q} 1} \otimes P_{m}\right)$, and $M_{2}\left(P_{n_{T 1}} \otimes P_{m}\right)$ by purple, orange, pink, and green colour, respectively.

(a) $M_{1}\left(G_{1} \otimes_{S_{k}} G_{2}\right)=M_{1}\left(S_{1} G_{1}\right)\left[n_{G_{2}}+2 e_{G_{2}}\right]+8(2 k-1) e_{G_{1}} e_{G_{2}}+\left[M_{1}\left(G_{1}\right)+4 e_{G_{1}}\right]\left[M_{1}\left(G_{2}\right)+2 e_{G_{2}}\right]$

$$
+M_{1}\left(G_{2}\right)\left[4 e_{G_{1}}+n_{G_{1}}\right]+4(k-1) e_{G_{1}}\left[n_{2}+M_{1}\left(G_{2}\right)\right],
$$

(b) $M_{2}\left(G_{1} \otimes_{S_{k}} G_{2}\right)=\left[M_{2}\left(G_{1}\right)+4 e_{G_{1}}\right]\left[5 e_{G_{2}}+3 M_{1}\left(G_{2}\right)+2 M_{2}\left(G_{2}\right)+n_{G_{2}}\right]+M_{1}\left(G_{1}\right)\left[e_{G_{2}}+M_{1}\left(G_{2}\right)+M_{2}\left(G_{2}\right)\right]$

$$
\begin{aligned}
& \left.+4(k-1) e_{G_{1}}\left[6 e_{G_{2}}+3 M_{1}\left(G_{2}\right)+M_{2}\left(G_{2}\right)+n_{G_{2}}\right]+M_{2}\left(G_{2}\right)\right]\left[12 e_{G_{1}}+n_{G_{1}}\right] \\
& +8 e_{G_{1}} e_{G_{2}}+M_{1}\left(G_{2}\right)\left[14 e_{G_{1}}\right] .
\end{aligned}
$$

Proof. Let $d(p, z)=d_{G_{1} \otimes_{S_{S}} G_{2}}(p, z)$ be the degree of a vertex $(p, z)$ in the graph $G_{1} \otimes_{S_{k}} G_{2}$.

$$
\begin{aligned}
M_{1}\left(G_{1} \otimes_{S_{k}} G_{2}\right)= & \sum_{(p, z) \varepsilon V\left(G_{1} \otimes_{S_{k}} G_{2}\right)} d^{2}(p, z)=\sum_{\left(p_{1}, z_{1}\right)\left(p_{2}, z_{2}\right) \varepsilon E\left(G_{1} \otimes_{S_{k}} G_{2}\right)}\left[d\left(p_{1}, z_{1}\right)+d\left(p_{2}, z_{2}\right)\right] \\
= & \sum_{p \varepsilon V\left(G_{1}\right)} \sum_{z_{1} p_{2} \varepsilon E\left(G_{2}\right)}\left[d\left(p, z_{1}\right)+d\left(p, z_{2}\right)\right]+\sum_{z \varepsilon V\left(G_{2}\right)} \sum_{p_{1} p_{2} \varepsilon E\left(S_{k}\left(G_{1}\right)\right)}\left[d\left(p_{1}, z\right)+d\left(p_{2}, z\right)\right] \\
& +\sum_{p_{1} p_{2} \varepsilon E\left(S_{k}\left(G_{1}\right)\right)} \sum_{z_{1} z_{2} \varepsilon V\left(G_{2}\right)}\left[d\left(p_{1}, z_{1}\right)+d\left(p_{2}, z_{2}\right)\right]=\sum 1+\sum 2+\sum 3 .
\end{aligned}
$$




\section{Consider}

$$
\begin{aligned}
\sum 1= & \sum_{p \varepsilon V\left(G_{1}\right)} \sum_{z_{1} z_{2} \varepsilon E\left(G_{2}\right)}\left[d\left(p, z_{1}\right)+d\left(p, z_{2}\right)\right]=\sum_{p \varepsilon V(G)} \sum_{z_{1} z_{2} \varepsilon E(H)}\left[2 d(p)+d\left(z_{1}\right)+d\left(z_{2}\right)+d(p)\left(d\left(z_{1}\right)+d\left(z_{2}\right)\right)\right] \\
= & 4 e_{G_{1}} e_{G_{2}}+M_{1}\left(G_{2}\right) n_{G_{1}}+2 M_{1}\left(G_{2}\right) e_{G_{1}} \cdot \\
\sum 2= & \sum_{z \varepsilon V\left(G_{2}\right)} \sum_{p_{1} p_{2} \varepsilon E\left(S_{k}\left(G_{1}\right)\right)}\left[d\left(p_{1}, y\right)+d\left(p_{2}, z\right)\right]=\sum_{z \varepsilon V\left(G_{2}\right) p_{1} \varepsilon V\left(G_{1}\right), p_{2} \varepsilon V\left(S_{k}\left(G_{1}\right)-G_{1}\right)}\left[d\left(p_{1}, z\right)+d\left(p_{2}, z\right)\right] \\
& +\sum_{z \varepsilon V\left(G_{2}\right) p_{1}, p_{2} \varepsilon V\left(S_{k}\left(G_{1}\right)-G_{1}\right)}\left[d\left(p_{1}, z\right)+d\left(p_{2}, z\right)\right]=\sum 2^{\prime}+\sum 2^{\prime \prime} \\
\sum 2^{\prime}= & \sum_{z \varepsilon V\left(G_{2}\right)} \sum_{p_{1} p_{2} \varepsilon E\left(S_{k}\left(G_{1}\right)\right) p_{1} \varepsilon V\left(G_{1}\right), p_{2} \varepsilon V\left(s_{k}\left(G_{1}\right)\right)-V\left(G_{1}\right)} \sum_{z \varepsilon V\left(G_{2}\right) p_{1} p_{2} \varepsilon E\left(S_{k}\left(G_{1}\right)\right) p_{1} \varepsilon V\left(G_{1}\right), p_{2} \varepsilon V\left(s_{k}\left(G_{1}\right)\right)-V\left(G_{1}\right)}\left[\left(d\left(p_{1}\right)+d\left(p_{2}\right)\right)+d(z)+\left(d\left(p_{1}\right)+d\left(p_{2}\right)\right) d(z)\right] .
\end{aligned}
$$

Since in this case $\left|E\left(S_{1}(G)\right)\right|=2|E(G)|$, we have

$$
\begin{aligned}
& =M_{1}\left(S_{1}\left(G_{1}\right)\right) n_{G_{2}}+4 e_{G_{1}} e_{G_{2}}+2 M_{1}\left(S_{1}\left(G_{1}\right)\right) e_{G_{2}}, \\
\sum 2^{\prime \prime} & \sum_{z \mathcal{E} V(G 2)}\left[d\left(p_{1}, z\right)+d\left(p_{2}, z\right)\right] \\
& =\sum_{z \mathcal{E} V(G 2)} \sum_{p_{1} p_{1} \varepsilon E\left(s_{2} \varepsilon E\left(S_{k}(G 1)\right) p_{1}, p_{2} \varepsilon V\left(S_{k}(G 1)\right)-V(G 1)\right.}\left[d\left(p_{1}\right)+d\left(p_{1}\right) d(z)+d\left(p_{2} \varepsilon V\left(s_{k}(G 1)\right)-V(G 1)\right.\right.
\end{aligned}
$$

Since in this case $\left|E\left(S_{k}(G 1)\right)\right|=(k-1)|E(G 1)|$, we have

$$
\begin{aligned}
= & \sum_{z \varepsilon V(G 2)}\left(4(k-1) e_{G_{1}}+4 d(z)(k-1) e_{G_{1}}\right)=4(k-1) e_{G_{1}} n_{G_{2}}+8 e_{G_{1}}(k-1) e_{G_{2}}, \\
\sum 3= & \sum_{p_{1} p_{2} \varepsilon E\left(S_{k}\left(G_{1}\right)\right)} \sum_{z_{1} z_{2} \varepsilon V\left(G_{2}\right)}\left[d\left(p_{1}, z_{1}\right)+d\left(p_{2}, z_{2}\right)\right] \\
= & \sum_{z_{1} z_{2} \varepsilon V\left(G_{2}\right) p_{1} p_{2} \varepsilon E\left(s_{k}\left(G_{1}\right)\right) p_{1} \varepsilon V\left(G_{1}\right), p_{2} \varepsilon V\left(s_{k}\left(G_{1}\right)\right)-V\left(G_{1}\right)}\left[d\left(p_{1}, z_{1}\right)+d\left(p_{2}, z_{2}\right)\right] \\
& +\sum_{z_{1} z_{2} \varepsilon V\left(G_{2}\right) p_{1} p_{2} \varepsilon E\left(s_{k}\left(G_{1}\right)\right) p_{1}, p_{2} \varepsilon V\left(s_{k}\left(G_{1}\right)\right)-V\left(G_{1}\right)}\left[d\left(p_{1}, z_{1}\right)+d\left(p_{2}, z_{2}\right)\right]=\sum 3^{\prime}+\sum 3^{\prime \prime}, \\
\sum 3^{\prime}= & \sum_{z_{1} z_{2} \varepsilon V\left(G_{2}\right) p_{1} p_{2} \varepsilon E\left(s_{k}\left(G_{1}\right)\right) p_{1} \varepsilon V\left(G_{1}\right), p_{2} \varepsilon V\left(s_{k}\left(G_{1}\right)\right)-V\left(G_{1}\right)}\left[d\left(p_{1}, z_{1}\right)+d\left(p_{2}, z_{2}\right)\right] \\
= & \sum_{z_{1} z_{2} \varepsilon V\left(G_{2}\right) p_{1} \varepsilon V\left(G_{1}\right), p_{2} \varepsilon V\left(s_{k}\left(G_{1}\right)\right)-V\left(G_{1}\right)}\left[\left(d\left(p_{1}\right)+d\left(p_{2}\right)\right)+d\left(z_{1}\right)+d\left(p_{1}\right) d\left(z_{1}\right)+d\left(p_{2}\right) d\left(z_{2}\right)\right] \\
= & 2 e_{G_{2}}\left[M_{1}\left(G_{1}\right)+4 e_{G_{1}}\right]+2 e_{G_{1}} M_{1}\left(G_{2}\right)+M_{1}\left(G_{2}\right)\left[M_{1}\left(G_{1}\right)+4 e_{G_{1}}\right], \\
\sum 3^{\prime \prime}= & \sum_{z_{1} z_{2} \varepsilon V\left(G_{2}\right) p_{1} p_{2} \varepsilon E\left(s_{k}\left(G_{1}\right)\right) p_{1}, p_{2} \varepsilon V\left(s_{k}\left(G_{1}\right)\right)-V\left(G_{1}\right)}\left[d\left(p_{1}, z_{1}\right)+d\left(p_{2}, z_{2}\right)\right] \\
= & \sum_{z_{1} z_{2} \varepsilon V\left(G_{2}\right) p_{1} p_{2} \varepsilon E\left(s_{k}\left(G_{1}\right)\right) p_{1}, p_{2} \varepsilon V\left(s_{k}\left(G_{1}\right)\right)-V\left(G_{1}\right)}\left[4+2\left(d\left(z_{1}\right)+d\left(z_{2}\right)\right)\right]=8(k-1) e_{G_{1}} e_{G_{2}}+4(k-1) e_{G_{1}} M_{1}\left(G_{2}\right) .
\end{aligned}
$$


Consequently, we get

$$
\begin{aligned}
M_{1}\left(G_{1} \otimes_{S_{k}} G_{2}\right)= & M_{1}\left(S_{1} G_{1}\right)\left[n_{G_{2}}+2 e_{G_{2}}\right]+8(2 k-1) e_{G_{1}} e_{G_{2}}+\left[M_{1}\left(G_{1}\right)+4 e_{G_{1}}\right]\left[M_{1}\left(G_{2}\right)+2 e_{G_{2}}\right] \\
& +M_{1}\left(G_{2}\right)\left[4 e_{G_{1}}+n_{G_{1}}\right]+4(k-1) e_{G_{1}}\left[n_{2}+M_{1}\left(G_{2}\right)\right], \\
(\mathbf{b}) M_{2}\left(G_{1} \otimes_{S_{k}} G_{2}\right)= & \sum_{(p, z) \varepsilon V\left(G_{1} \bigotimes_{S_{k}} G_{2}\right)} d^{2}(p, z)=\sum_{\left(p_{1}, z_{1}\right)\left(p_{2}, z_{2}\right) \varepsilon E\left(G_{1} \otimes_{S_{k}} G_{2}\right)}\left[d\left(p_{1}, z_{1}\right) d\left(p_{2}, z_{2}\right)\right] \\
= & \sum_{p \varepsilon V\left(G_{1}\right)} \sum_{z_{1} z_{2} \varepsilon E\left(G_{2}\right)}\left[d\left(p, z_{1}\right) d\left(p, z_{2}\right)\right]+\sum_{z \varepsilon V\left(G_{2}\right)} \sum_{p_{1} p_{2} \varepsilon E\left(S_{k}\left(G_{1}\right)\right)}\left[d\left(p_{1}, z\right) d\left(p_{2}, z\right)\right] \\
& +\sum_{p_{1} p_{2} \varepsilon E\left(S_{k}\left(G_{1}\right)\right) z_{1} z_{2} \varepsilon V\left(G_{2}\right)}\left[d\left(p_{1}, z_{1}\right) d\left(p_{2}, z_{2}\right)\right]=\sum 1+\sum 2+\sum 3 .
\end{aligned}
$$

Consider

$$
\begin{aligned}
& \sum 1=\sum_{p \varepsilon V\left(G_{1}\right)} \sum_{z_{1} z_{2} \varepsilon E\left(G_{2}\right)}\left[d\left(p, z_{1}\right) d\left(p, z_{2}\right)\right]=\sum_{p \varepsilon V\left(G_{1}\right)} \sum_{z_{1} z_{2} \varepsilon E\left(G_{2}\right)}\left[d(p)+d\left(z_{1}\right)\right]\left[d(p)+d\left(z_{2}\right)\right] \\
& =\sum_{p \varepsilon V\left(G_{1}\right)} \sum_{z_{1} z_{2} \varepsilon E\left(G_{2}\right)}\left[d^{2}(p)+d^{2}(p)\left(d\left(z_{1}+d\left(z_{2}\right)\right)+d(p)\left(d\left(z_{1}\right)+d\left(z_{2}\right)+2 d\left(z_{1}\right) d\left(z_{2}\right)\right)+d\left(z_{1}\right) d\left(z_{2}\right)\right]\right. \\
& =M_{1}\left(G_{1}\right) e_{G_{2}}+M_{1}\left(G_{1}\right)\left[M_{1}\left(G_{2}\right)+M_{2}\left(G_{2}\right)\right]+2 e_{G_{1}}\left[M_{1}\left(G_{2}\right)+2 M_{2}\left(G_{2}\right)\right]+M_{2}\left(G_{2}\right) n_{G_{1}}, \\
& \sum 2=\sum_{z \varepsilon V\left(G_{2}\right)} \sum_{p_{1} p_{2} \varepsilon E\left(S_{k}\left(G_{1}\right)\right) p_{1} \varepsilon V\left(G_{1}\right), p_{2} \varepsilon V\left(S_{k}\left(G_{1}\right)-G_{1}\right)}\left[d\left(p_{1}, z\right) d\left(p_{2}, z\right)\right] \\
& +\sum_{z \varepsilon V\left(G_{2}\right)} \sum_{p_{1} p_{2} \varepsilon E\left(S_{k}\left(G_{1}\right)\right)}\left[d\left(p_{1}, p_{2} \varepsilon V\left(S_{k}\left(G_{1}\right)-G_{1}\right) d\left(p_{2}, z\right)\right]\right. \\
& =\sum 2^{\prime}+\sum 2^{\prime \prime} \\
& \sum 2^{\prime}=\sum_{z \varepsilon V\left(G_{2}\right)} \sum_{p_{1} p_{2} \varepsilon E\left(S_{k}\left(G_{1}\right)\right) p_{1} \varepsilon V\left(G_{1}\right), p_{2} \varepsilon V\left(S_{k}\left(G_{1}\right)\right)-V\left(G_{1}\right)}\left[d\left(p_{1}, z\right) d\left(p_{2}, z\right)\right] \\
& =\sum_{z \varepsilon V\left(G_{2}\right)} \sum_{p_{1} p_{2} \varepsilon E\left(S_{k}\left(G_{1}\right)\right) p_{1} \varepsilon V\left(G_{1}\right), p_{2} \varepsilon V\left(S_{k}\left(G_{1}\right)\right)-V\left(G_{1}\right)}\left[\left(d\left(p_{1}\right)+d(z)+d\left(p_{1}\right) d(z)\right)\left(d\left(p_{2}\right)+d\left(p_{2}\right) d(z)\right)\right] \\
& =\sum_{z \varepsilon V\left(G_{2}\right)} \sum_{p_{1} p_{2} \varepsilon E\left(S_{k}\left(G_{1}\right)\right) p_{1} \varepsilon V\left(G_{1}\right), p_{2} \varepsilon V\left(S_{k}\left(G_{1}\right)\right)-V\left(G_{1}\right)}\left[d\left(p_{1}\right) d\left(p_{2}\right)+2 d\left(p_{1}\right) d\left(p_{2}\right) d(z)+d\left(p_{2}\right) d(y)+d\left(p_{2}\right) d^{2}(z)\right. \\
& \left.+d\left(p_{1}\right) d\left(p_{2}\right) d^{2}(z)\right] \\
& =\left[M_{2}\left(G_{1}\right)+4 e_{G_{1}}\right]\left[n_{G_{2}}+4 e_{G_{2}}+M_{1}\left(G_{2}\right)\right]+8 e_{G_{1}} e_{G_{2}}+4 e_{G_{1}} M_{1}\left(G_{2}\right), \\
& \sum 2^{\prime \prime}=\sum_{z \varepsilon V\left(G_{2}\right)} \sum_{p_{1} p_{2} \varepsilon E\left(S_{k}\left(G_{1}\right)\right) p_{1}, p_{2} \varepsilon V\left(S_{k}\left(G_{1}\right)\right)-V\left(G_{1}\right)}\left[d\left(p_{1}, z\right) d\left(p_{2}, z\right)\right] \\
& =\sum_{z \varepsilon V\left(G_{2}\right)} \sum_{p_{1} p_{2} \varepsilon E\left(S_{k}\left(G_{1}\right)\right)} \sum_{p_{1}, p_{2} \varepsilon V\left(S_{k}\left(G_{1}\right)\right)-V\left(G_{1}\right)}\left[\left(d\left(p_{1}\right)+d\left(p_{1}\right) d(z)\right)\left(d\left(p_{2}\right)+d\left(p_{2}\right) d(z)\right)\right] \\
& =\sum_{z \varepsilon V\left(G_{2}\right)} \sum_{p_{1} p_{2} \varepsilon E\left(S_{k}\left(G_{1}\right)\right) p_{1} \varepsilon V\left(G_{1}\right), p_{2} \varepsilon V\left(S_{k}\left(G_{1}\right)\right)-V\left(G_{1}\right)}\left[4+8 d(z)+4 d^{2}(z)\right]=4(k-1) e_{G_{1}}\left[n_{G_{2}}+4 e_{G_{2}}+M_{1}\left(G_{2}\right)\right], \\
& \sum 3=\sum_{z_{1} z_{2} \varepsilon V\left(G_{2}\right)} \sum_{p_{1} p_{2} \varepsilon E\left(S_{k}\left(G_{1}\right)\right) p_{1} \varepsilon V\left(G_{1}\right), p_{2} \varepsilon V\left(S_{k}\left(G_{1}\right)\right)-V\left(G_{1}\right)}\left[d\left(p_{1}, z_{1}\right) d\left(p_{2}, z_{2}\right)\right] \\
& +\sum_{z_{1} z_{2} \varepsilon V\left(G_{2}\right)} \sum_{p_{1} p_{2} \varepsilon E\left(S_{k}\left(G_{1}\right)\right)}\left[d\left(p_{1}, z_{1}\right) d\left(p_{2}, z_{2}\right)\right]=\sum 3^{\prime}+\sum 3^{\prime \prime},
\end{aligned}
$$




$$
\begin{aligned}
& \sum 3^{\prime}=\sum_{z_{1} z_{2} \varepsilon V\left(G_{2}\right)} \sum_{p_{1} p_{2} \varepsilon E\left(S_{k}\left(G_{1}\right)\right) p_{1} \varepsilon V\left(G_{1}\right), p_{2} \varepsilon V}\left[d\left(s_{k}\left(G_{1}\right)\right)-V\left(G_{1}\right) d\left(p_{2}, z_{2}\right)\right] \\
& \left.=\sum_{z_{1} z_{2} \varepsilon V\left(G_{2}\right) p_{1} p_{2} \varepsilon E\left(S_{k}\left(G_{1}\right)\right) p_{1} \varepsilon V\left(G_{1}\right), p_{2} \varepsilon V\left(S_{k}\left(G_{1}\right)\right)-V\left(G_{1}\right)}\left(d\left(p_{1}\right)+d\left(z_{1}\right)+d\left(p_{1}\right) d\left(z_{1}\right)\right)\left(d\left(p_{2}\right)+d\left(p_{2}\right) d\left(z_{2}\right)\right)\right] \\
& =\sum_{z \varepsilon V\left(G_{2}\right)} \sum_{p_{1} p_{2} \varepsilon E\left(S_{k}\left(G_{1}\right)\right) p_{1} \varepsilon V\left(G_{1}\right), p_{2} \varepsilon V\left(S_{k}\left(G_{1}\right)\right)-V\left(G_{1}\right)} \\
& \cdot\left[d\left(p_{1}\right) d\left(p_{2}\right)+d\left(p_{1}\right) d\left(p_{2}\right) d\left(z_{2}\right)+d\left(p_{2}\right) d\left(z_{1}\right)+d\left(p_{1}\right) d\left(z_{1}\right) d\left(z_{2}\right)+d\left(p_{1}\right) d\left(p_{2}\right) d\left(z_{1}\right)+d\left(p_{1}\right) d\left(p_{2}\right) d\left(z_{1}\right) d\left(z_{2}\right)\right] \\
& =\left[M_{2}\left(G_{1}\right)+4 e_{G_{1}}\right]\left[e_{G_{2}}+2 M_{1}\left(G_{2}\right)+2 M_{2}\left(G_{2}\right)\right]+8 e_{G_{1}} M_{1}\left(G_{2}\right)+8 e_{G_{1}} M_{2}\left(G_{2}\right) \text {. } \\
& \sum 3^{\prime \prime}=\sum_{z \varepsilon V(G 2)} \sum_{p_{1} p_{2} \varepsilon E\left(S_{k}(G 1)\right) z_{1}, p_{2} \varepsilon V\left(s_{k}(G 1)\right)-V(G 1)}\left[d\left(p_{1}, z_{1}\right) d\left(p_{2}, z_{2}\right)\right]
\end{aligned}
$$

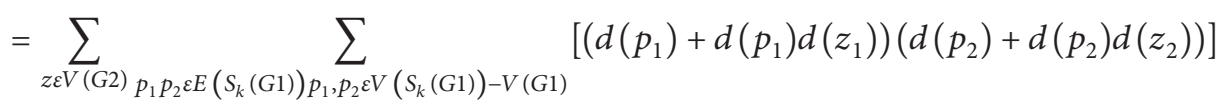

$$
\begin{aligned}
& =\sum_{z \varepsilon V\left(G_{2}\right) p_{1} \varepsilon V\left(G_{1}\right), p_{2} \varepsilon V\left(S_{k}\left(G_{1}\right)\right)-V\left(G_{1}\right)}\left[4+4\left(d\left(z_{2}\right)+d\left(z_{1}\right)\right)+4 d\left(z_{1}\right) d\left(z_{2}\right)\right]=8(k-1) e_{G_{1}}\left[e_{G_{2}}+M_{1}\left(G_{2}\right)+M_{2}\left(G_{2}\right)\right] .
\end{aligned}
$$

Therefore,

$$
\begin{aligned}
M_{2}\left(G_{1} \unrhd_{S_{k}} G_{2}\right)= & {\left[M_{2}\left(G_{1}\right)+4 e_{G_{1}}\right]\left[5 e_{G_{2}}+3 M_{1}\left(G_{2}\right)+2 M_{2}\left(G_{2}\right)+n_{G_{2}}\right]+M_{1}\left(G_{1}\right)\left[e_{G_{2}}+M_{1}\left(G_{2}\right)+M_{2}\left(G_{2}\right)\right] } \\
& \left.+4(k-1) e_{G_{1}}\left[6 e_{G_{2}}+3 M_{1}\left(G_{2}\right)+M_{2}\left(G_{2}\right)+n_{G_{2}}\right]+M_{2}\left(G_{2}\right)\right]\left[12 e_{G_{1}}+n_{G_{1}}\right] \\
& +8 e_{G_{1}} e_{G_{2}}+M_{1}\left(G_{2}\right)\left[14 e_{G_{1}}\right] .
\end{aligned}
$$

Theorem 2. Let $G_{1}$ and $G_{2}$ be two connected graphs such that $\left|V\left(G_{1}\right)\right|,\left|V\left(G_{2}\right)\right| \geq 4$. For $k \geq 1$,

$$
\text { (a) } \begin{aligned}
M_{1}\left(G_{1} \unrhd_{R_{k}} G_{2}\right)= & M_{1}\left(G_{2}\right)\left[n_{G_{1}}+12 e_{G_{1}}+4 M_{1}\left(G_{1}\right)\right]+M_{1}\left(G_{1}\right)\left[12 e_{G_{2}}+2 n_{G_{2}}\right]+24 e_{G_{1}} e_{G_{2}} \\
& +\left[M_{1}\left(R_{1}\left(G_{1}\right)\right)-2 M_{1} G_{1}\right]\left[n_{G_{2}}+2 e_{G_{2}}\right]+4(k-1) e_{G_{1}}\left[n_{G_{2}}+4 e_{G_{2}}+M_{1}\left(G_{2}\right)\right], \\
\text { (b) } M_{2}\left(G_{1} \bigotimes_{R_{k}} G_{2}\right)= & M_{1}\left(G_{1}\right)\left[8 e_{G_{2}}+12 M_{1}\left(G_{2}\right)+4 M_{2}\left(G_{2}\right)\right]+4 M_{2}\left(G_{1}\right)\left[n_{G_{2}}+6 e_{G_{2}}+3 M_{1}\left(G_{2}\right)\right] \\
& +\left[M_{2}\left(R_{1}\left(G_{1}\right)\right)-4 M_{2} G_{1}+4(k-1) e_{G_{1}}\right]\left[6 e_{G_{2}}+3 M_{1}\left(G_{2}\right)+2 M_{2}\left(G_{2}\right)+n_{G_{2}}\right] \\
& +M_{2}\left(G_{2}\right)\left[n_{G_{1}}+2 e_{G_{1}}\right]+4 e_{G_{1}}\left[3 M_{1}\left(G_{2}\right)+3 M_{2}\left(G_{2}\right)+4 e_{G_{2}}\right]+e_{G_{1}} M_{1}\left(G_{2}\right) .
\end{aligned}
$$


Proof. Let $d(p, z)=d_{G_{1} \otimes_{R} G_{2}}(p, z)$ be the degree of a vertex $(p, z)$ in the graph $G_{1} \otimes_{R_{k}} G_{2}$.

$$
\begin{aligned}
& M_{1}\left(G_{1} \otimes_{R_{k}} G_{2}\right)=\sum_{(p, z) \varepsilon V\left(G_{1} \otimes_{R_{k}} G_{2}\right)} d^{2}(x, z)=\sum_{\left(p_{1}, z_{1}\right)} \sum_{\left(p_{2}, z_{2}\right) \varepsilon E\left(G_{1} \otimes_{R_{k}} G_{2}\right)}\left[d\left(p_{1}, z_{1}\right)+d\left(z_{2}, z_{2}\right)\right] \\
& =\sum_{p \varepsilon V\left(G_{1}\right)} \sum_{z_{1} z_{2} \varepsilon E\left(G_{2}\right)}\left[d\left(p, z_{1}\right)+d\left(p, z_{2}\right)\right]+\sum_{z \varepsilon V\left(G_{2}\right)} \sum_{p_{1} p_{2} \varepsilon E\left(R_{k}\left(G_{1}\right)\right)}\left[d\left(p_{1}, z\right)+d\left(p_{2}, z\right)\right] \\
& +\sum_{p_{1} p_{2} \varepsilon E\left(R_{k}\left(G_{1}\right)\right)} \sum_{z_{1} z_{2} \varepsilon V\left(G_{2}\right)}\left[d\left(p_{1}, z_{1}\right)+d\left(p_{2}, z_{2}\right)\right]=\sum 1+\sum 2+\sum 3, \\
& \sum 1=\sum_{p \varepsilon V\left(G_{1}\right)} \sum_{z_{1} z_{2} \varepsilon E\left(G_{2}\right)}\left[d\left(p, y_{1}\right)+d\left(p, y_{2}\right)\right] \\
& =\sum_{p \varepsilon V\left(G_{1}\right)} \sum_{z_{1} z_{2} \varepsilon E\left(G_{2}\right)}\left[2 d(p)+d\left(z_{1}\right)+2 d(p) d\left(z_{1}\right)+2 d(p)+d\left(z_{2}\right)+2 d(p) d\left(z_{2}\right)\right] \\
& =\sum_{p \varepsilon V\left(G_{1}\right)} \sum_{z_{1} z_{2} \varepsilon E\left(G_{2}\right)}\left[4 d(p)+d\left(z_{1}\right)+d\left(z_{2}\right)+2 d(p)\left(d\left(z_{1}\right)+d\left(z_{2}\right)\right)\right] \\
& =8 e_{G_{1}} e_{G_{2}}+M_{1}\left(G_{2}\right) n_{G_{1}}+4 M_{1}\left(G_{2}\right) e_{G_{1}}, \\
& \sum 2=\sum_{z \varepsilon V\left(G_{2}\right)} \sum_{p_{1} p_{2} \varepsilon E\left(R_{k}\left(G_{1}\right)\right)}\left[d\left(p_{1}, z\right)+d\left(p_{2}, z\right)\right]=\sum_{z \varepsilon V\left(G_{2}\right)} \sum_{p_{1} p_{2} \varepsilon E\left(R_{k}\left(G_{1}\right)\right) p_{1}, p_{2} \varepsilon V\left(G_{1}\right)}\left[d\left(p_{1}, z\right)+d\left(p_{2}, z\right)\right] \\
& +\sum_{z \varepsilon V\left(G_{2}\right)} \sum_{p_{1} p_{2} \varepsilon E\left(R_{k}\left(G_{1}\right)\right) p_{1} \varepsilon V\left(G_{1}\right), p_{2} \varepsilon V\left(R_{k}\left(G_{1}\right)-G_{1}\right)}\left[d\left(p_{1}, y\right)+d\left(p_{2}, z\right)\right]+\sum_{z \varepsilon V\left(G_{2}\right)} \sum_{p_{1} p_{2} \varepsilon E\left(R_{k}\left(G_{1}\right)\right) p_{1}, p_{2} \varepsilon V\left(R_{k}\left(G_{1}\right)-G_{1}\right)} \\
& \cdot\left[d\left(p_{1}, z\right)+d\left(p_{2}, y\right)\right] \\
& =\sum 2^{\prime}+\sum 2^{\prime \prime}+\sum 2^{\prime \prime \prime} \\
& \sum 2^{\prime}=\sum_{z \varepsilon V\left(G_{2}\right)} \sum_{p_{1} p_{2} \varepsilon E\left(R_{k}\left(G_{1}\right)\right) p_{1}, p_{2} \varepsilon V\left(G_{1}\right)}\left[d\left(p_{1}, z\right)+d\left(p_{2}, z\right)\right] \\
& =\sum_{z \varepsilon V\left(G_{2}\right)} \sum_{p_{1} p_{2} \varepsilon E\left(R_{k}\left(G_{1}\right)\right) p_{1}, p_{2} \varepsilon V\left(G_{1}\right)}\left[d\left(p_{1}\right)+d(z)+d\left(p_{1}\right) d(z)+d\left(p_{2}\right)+d(z)+d\left(p_{2}\right) d(z)\right] \\
& =\sum_{z \varepsilon V\left(G_{2}\right)} \sum_{p_{1} p_{2} \varepsilon E\left(R_{k}\left(G_{1}\right)\right) p_{1}, p_{2} \varepsilon V\left(G_{1}\right)}\left[d\left(p_{1}\right)+d\left(p_{2}\right)+2 d(z)+d(y)\left(d\left(p_{1}\right)+d\left(p_{2}\right)\right)\right] \\
& =2 n_{G_{2}} M_{1}\left(G_{1}\right)+4 e_{G_{1}} e_{G_{2}}+4 e_{G_{2}} M_{1}\left(G_{1}\right) \text {, } \\
& \sum 2^{\prime \prime}=\sum_{z \varepsilon V\left(G_{2}\right)} \sum_{p_{1} p_{2} \varepsilon E\left(R_{k}\left(G_{1}\right)\right) p_{1} \varepsilon V\left(G_{1}\right), p_{2} \varepsilon V\left(S_{k}\left(G_{1}\right)\right)-V\left(G_{1}\right)}\left[d\left(p_{1}, z\right)+d\left(p_{2}, y\right)\right] \\
& =\sum_{z \varepsilon V\left(G_{2}\right) p_{1} p_{2} \varepsilon E\left(R_{k}\left(G_{1}\right)\right) p_{1} \varepsilon V\left(G_{1}\right), p_{2} \varepsilon V\left(S_{k}\left(G_{1}\right)\right)-V\left(G_{1}\right)}\left[d\left(p_{1}\right)+d\left(p_{2}\right)+d(z)+\left(d\left(p_{1}\right)+d\left(p_{2}\right)\right) d(z)\right] \\
& =\left[M_{1}\left(R_{1}\left(G_{1}\right)\right)-2 M_{1}\left(G_{1}\right)\right]\left[n_{G_{2}}+2 e_{G_{2}}\right]+4 e_{G_{1}} e_{G_{2}}, \\
& \sum 2^{\prime \prime \prime}=\sum_{z \varepsilon V(G 2)} \sum_{p_{1} p_{2} \varepsilon E\left(R_{k}(G 1)\right) p_{1}, p_{2} \varepsilon V\left(R_{k}(G 1)\right)-V(G 1)}\left[d\left(p_{1}, z\right)+d\left(p_{2}, z\right)\right] \\
& =\sum_{z \varepsilon V(G 2)} \sum_{p_{1} p_{2} \varepsilon E\left(R_{k}(G 1)\right) p_{1}, p_{2} \varepsilon V\left(R_{k}(G 1)\right)-V(G 1)}\left[d\left(p_{1}\right)+d\left(p_{2}\right)+\left(d\left(p_{1}\right)+d\left(p_{2}\right)\right) d(z)\right] \\
& =4(k-1) e_{G_{1}} n_{G_{2}}+8(k-1) e_{G_{1}} e_{G_{2}}, \\
& \sum 3=\sum_{p_{1} p_{2} \varepsilon E\left(R_{k}\left(G_{1}\right)\right)} \sum_{z_{1} z_{2} \varepsilon V\left(G_{2}\right)}\left[d\left(p_{1}, z_{1}\right)+d\left(p_{2}, z_{2}\right)\right]=\sum_{z_{1} z_{2} \varepsilon V\left(G_{2}\right)} \sum_{p_{1} p_{2} \varepsilon E\left(R_{k}\left(G_{1}\right)\right) p_{1}, p_{2} \varepsilon V\left(G_{1}\right)}\left[d\left(p_{1}, z_{1}\right)+d\left(p_{2}, z_{2}\right)\right] \\
& +\sum_{z_{1} z_{2} \varepsilon V\left(G_{2}\right)} \sum_{p_{1} p_{2} \varepsilon E\left(R_{k}\left(G_{1}\right)\right) p_{1} \varepsilon V\left(G_{1}\right), p_{2} \varepsilon V\left(R_{k}\left(G_{1}\right)\right)-V\left(G_{1}\right)}\left[d\left(p_{1}, z_{1}\right)+d\left(p_{2}, z_{2}\right)\right] \\
& +\sum_{z_{1} z_{2} \varepsilon V\left(G_{2}\right)} \sum_{p_{1} p_{2} \varepsilon E\left(R_{k}\left(G_{1}\right)\right) p_{1}, p_{2} \varepsilon V\left(R_{k}\left(G_{1}\right)\right)-V\left(G_{1}\right)}\left[d\left(p_{1}, z_{1}\right)+d\left(p_{2}, z_{2}\right)\right]=\sum 3^{\prime}+\sum 3^{\prime \prime}+\sum 3^{\prime \prime \prime},
\end{aligned}
$$




$$
\begin{aligned}
& \sum 3^{\prime}=\sum_{z_{1} z_{2} \varepsilon V\left(G_{2}\right)} \sum_{p_{1} p_{2} \varepsilon E} \sum_{\left(R_{k}\left(G_{1}\right)\right)}\left[d\left(p_{1}, p_{2} \varepsilon V\left(z_{1}\right)+d\left(p_{2}, z_{2}\right)\right]\right. \\
& =\sum_{z_{1} z_{2} \varepsilon V\left(G_{2}\right)} \sum_{\left.p_{1} p_{2} \varepsilon E\left(R_{k}\left(G_{1}\right)\right)\right)}\left[d\left(p_{1}\right)+d\left(p_{2} \varepsilon V\left(G_{1}\right)+d\left(p_{1}\right) d\left(z_{1}\right)+d\left(p_{2}\right)+d\left(z_{2}\right)+d\left(p_{2}\right) d\left(z_{2}\right)\right]\right. \\
& =\sum_{z_{1} z_{2} \varepsilon V\left(G_{2}\right)} \sum_{p_{1} p_{2} \varepsilon E\left(R_{k}\left(G_{1}\right)\right) p_{1}, p_{2} \varepsilon V\left(G_{1}\right)}\left[d\left(p_{1}\right)+d\left(p_{2}\right)+d\left(z_{1}\right)+d\left(z_{2}\right)+d\left(p_{1}\right) d\left(z_{1}\right)+d\left(p_{2}\right) d\left(z_{2}\right)\right] \\
& =4 e_{G_{2}} M_{1}\left(G_{1}\right)+2 M_{1}\left(G_{2}\right) e_{G_{1}}+2 M_{1}\left(G_{2}\right) M_{1}\left(G_{1}\right) \text {, } \\
& \sum 3^{\prime \prime}=\sum_{z_{1} z_{2} \varepsilon V\left(G_{2}\right)} \sum_{p_{1} p_{2} \varepsilon E\left(R_{k}\left(G_{1}\right)\right) p_{1} \varepsilon V\left(G_{1}\right), p_{2} \varepsilon V\left(R_{k}\left(G_{1}\right)\right)-V\left(G_{1}\right)}\left[d\left(p_{1}, z_{1}\right)+d\left(p_{2}, z_{2}\right)\right] \\
& =\sum_{z_{1} z_{2} \varepsilon V\left(G_{2}\right)} \sum_{p_{1} p_{2} \varepsilon E\left(R_{k}\left(G_{1}\right)\right) p_{1} \varepsilon V\left(G_{1}\right), p_{2} \varepsilon V\left(R_{k}\left(G_{1}\right)\right)-V\left(G_{1}\right)}\left[d\left(p_{1}\right)+d\left(z_{1}\right)+d\left(p_{1}\right) d\left(z_{1}\right)+d\left(p_{2}\right)+d\left(p_{2} z_{2}\right)\right] \\
& =4 e_{G_{2}} M_{1}\left(G_{1}\right)+2 M_{1}\left(G_{2}\right) e_{G_{1}}+2 M_{1}\left(G_{2}\right) M_{1}\left(G_{1}\right)+8 e_{G_{1}} e_{G_{2}}+4 e_{G_{1}} M_{1}\left(G_{2}\right), \\
& \sum 3^{\prime \prime \prime}=\sum_{z_{1} z_{2} \varepsilon V\left(G_{2}\right)} \sum_{p_{1} p_{2} \varepsilon E\left(R_{k}\left(G_{1}\right)\right) p_{1}, p_{2} \varepsilon V\left(R_{k}\left(G_{1}\right)\right)-V\left(G_{1}\right)}\left[d\left(p_{1}, z_{1}\right)+d\left(p_{2}, z_{2}\right)\right]
\end{aligned}
$$

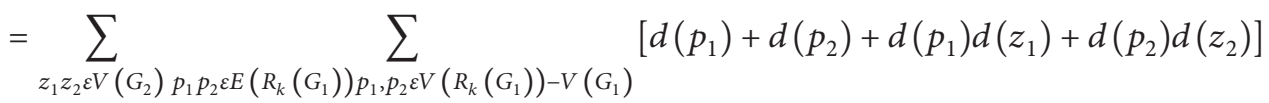

$$
\begin{aligned}
& =8(k-1) e_{G_{1}} e_{G_{2}}+4(k-1) e_{G_{1}} M_{1}\left(G_{2}\right) .
\end{aligned}
$$

Hence,

$$
\begin{aligned}
M_{1}\left(G_{1} \otimes_{R_{k}} G_{2}\right)= & M_{1}\left(G_{2}\right)\left[n_{G_{1}}+12 e_{G_{1}}+4 M_{1}\left(G_{1}\right)\right]+M_{1}\left(G_{1}\right)\left[12 e_{G_{2}}+2 n_{G_{2}}\right]+24 e_{G_{1}} e_{G_{2}} \\
& +\left[M_{1}\left(R_{1}\left(G_{1}\right)\right)-2 M_{1} G_{1}\right]\left[n_{G_{2}}+2 e_{G_{2}}\right]+4(k-1) e_{G_{1}}\left[n_{G_{2}}+4 e_{G_{2}}+M_{1}\left(G_{2}\right)\right], \\
(\mathbf{b}) M_{2}\left(G_{1} \bigotimes_{R_{k}} G_{2}\right)= & \sum_{(x, z) \varepsilon V\left(G_{1} \otimes_{R_{k}} G_{2}\right)} d^{2}(p, z)=\sum_{\left(p_{1}, z_{1}\right)\left(p_{2}, z_{2}\right) \varepsilon E\left(G_{1} \otimes_{R_{k}} G_{2}\right)}\left[d\left(p_{1}, z_{1}\right) d\left(p_{2}, z_{2}\right)\right] \\
= & \sum_{p \varepsilon V\left(G_{1}\right)} \sum_{z_{1} z_{2} \varepsilon E\left(G_{2}\right)}\left[d\left(p, z_{1}\right) d\left(p, z_{2}\right)\right]+\sum_{z \varepsilon V\left(G_{2}\right)} \sum_{p_{1} p_{2} \varepsilon E\left(R_{k}\left(G_{1}\right)\right)}\left[d\left(p_{1}, z\right) d\left(p_{2}, z\right)\right] \\
& +\sum_{p_{1} p_{2} \varepsilon E\left(R_{k}\left(G_{1}\right)\right)} \sum_{z_{1} z_{2} \varepsilon V\left(G_{2}\right)}\left[d\left(p_{1}, z_{1}\right) d\left(p_{2}, z_{2}\right)\right]=\sum 1+\sum 2+\sum 3 .
\end{aligned}
$$


Consider

$$
\begin{aligned}
& \sum 1=\sum_{p \& V\left(G_{1}\right)} \sum_{z_{1} z_{2} E E\left(G_{2}\right)}\left[d\left(p, z_{1}\right) d\left(p, z_{2}\right)\right]=\sum_{p \& V\left(G_{1}\right)} \sum_{z_{1} z_{2} E E\left(G_{2}\right)}\left[d(p)+d\left(z_{1}\right)\right]\left[d(p)+d\left(z_{2}\right)\right] \\
& =\sum_{p \in V\left(G_{1}\right)} \sum_{z_{1} z_{2} E\left(G_{2}\right)}\left[d^{2}(p)+d^{2}(p)\left(d\left(z_{1}\right)+d\left(z_{2}\right)\right)+d^{2}(p)\left(d\left(z_{1}\right)+d\left(z_{2}\right)\right)+2 d(p) d\left(z_{1}\right) d\left(z_{2}\right)+d\left(z_{1}\right) d\left(z_{2}\right)\right. \\
& =\sum_{z_{1} z_{2} E E\left(G_{2}\right)}\left[4 M_{1}\left(G_{1}\right)+4 M_{1}\left(G_{1}\right)\right]\left[d\left(z_{1}\right)+d\left(z_{2}\right)+d\left(z_{1}\right) d\left(z_{2}\right)\right]+4 e_{G_{1}}\left[d\left(z_{1}\right)+d\left(z_{2}\right)+2 d\left(z_{1}\right) d\left(z_{2}\right)\right] \\
& +d\left(z_{1}\right) d\left(z_{2}\right)\left(n_{G_{1}}\right)=4 M_{1}\left(G_{1}\right)\left[e_{G_{2}}+M_{1}\left(G_{2}\right)+M_{2}\left(G_{2}\right)\right]+4 e_{G_{1}}\left[M_{1}\left(G_{2}\right)+2 M_{2}\left(G_{2}\right)\right]+M_{2}\left(G_{2}\right) n_{G_{1}}, \\
& \sum 2=\sum_{z \varepsilon V\left(G_{2}\right)} \sum_{p_{1} p_{2} \varepsilon E\left(R_{k}\left(G_{1}\right)\right)}\left[d\left(p_{1}, z\right) d\left(p_{2}, z\right)\right] \\
& =\sum_{z \varepsilon V\left(G_{2}\right)} \sum_{p_{1} p_{2} \varepsilon E\left(R_{k}\left(G_{1}\right)\right) p_{1}, p_{2} \varepsilon V\left(G_{1}\right)}\left[d\left(p_{1}, z\right) d\left(p_{2}, z\right)\right]+\sum_{z \in V\left(G_{2}\right)} \sum_{p_{1} p_{2} \varepsilon E\left(R_{k}\left(G_{1}\right)\right) p_{1} \varepsilon V\left(G_{1}\right), p_{2} \varepsilon V\left(R_{k}\left(G_{1}\right)-G_{1}\right)}\left[d\left(p_{1}, z\right) d\left(p_{2}, z\right)\right] \\
& +\sum_{z \varepsilon V\left(G_{2}\right)} \sum_{p_{1} p_{2} E E\left(R_{k}\left(G_{1}\right)\right) p_{1}, p_{2} E V\left(R_{k}\left(G_{1}\right)-G_{1}\right)}\left[d\left(p_{1}, z\right) d\left(p_{2}, z\right)\right]=\sum 2^{\prime}+\sum 2^{\prime \prime}+\sum 2^{\prime \prime \prime}, \\
& \sum 2^{\prime}=\sum_{z \varepsilon V\left(G_{2}\right)} \sum_{p_{1} p_{2} \varepsilon\left(R_{k}\left(G_{1}\right)\right)}\left[d\left(p_{1}, z\right) d\left(p_{2}, z\right)\right] \\
& =\sum_{z \in V\left(G_{2}\right)} \sum_{p_{1} p_{2} \varepsilon E\left(R_{k}\left(G_{1}\right)\right)}\left[d\left(p_{1}\right)+d(z)+d\left(p_{1}\right) d(z)\right]\left[d\left(p_{2}\right)+d(z)+d\left(p_{2}\right) d(z)\right] \\
& =\sum_{z \varepsilon V\left(G_{2}\right)} \sum_{p_{1} p_{2} \varepsilon E\left(R_{k}\left(G_{1}\right)\right)}\left[4 d\left(p_{1}\right) d\left(p_{2}\right)+2 d\left(p_{1}\right) d(z)+4 d\left(p_{1}\right) d\left(p_{2}\right) d(z)+2 d\left(p_{2}\right) d(z)\right. \\
& \left.+d^{2}(z)+2 d\left(p_{2}\right) d^{2}(z)+4 d\left(p_{1}\right) d\left(p_{2}\right) d(z)+4 d\left(p_{1}\right) d\left(p_{2}\right) d^{2}(z)\right] \\
& =4 M_{2}\left(G_{1}\right)\left[n_{G_{2}}+4 e_{G_{2}}+M_{1}\left(G_{2}\right)+2 M_{1}\left(G_{1}\right)\left[M_{1}\left(G_{2}\right)+2 e_{G_{2}}+e_{G_{1}} M_{1}\left(G_{2}\right),\right.\right. \\
& \sum 2^{\prime \prime}=\sum_{z \varepsilon V\left(G_{2}\right)} \sum_{p_{1} p_{2} \varepsilon E\left(R_{k}\left(G_{1}\right)\right) p_{1} \varepsilon V\left(G_{1}\right), p_{2} \varepsilon V\left(R_{k}\left(G_{1}\right)\right)-V\left(G_{1}\right)}\left[d\left(p_{1}, z\right) d\left(p_{2}, z\right)\right] \\
& =\sum_{z \varepsilon V\left(G_{2}\right)} \sum_{p_{1} p_{2} \varepsilon E\left(R_{k}\left(G_{1}\right)\right) p_{1} \varepsilon V\left(G_{1}\right), p_{2} \varepsilon V\left(R_{k}\left(G_{1}\right)\right)-V\left(G_{1}\right)}\left[\left(d\left(p_{1}+d(z)+d\left(p_{1}\right) d(z)\right)\left(d\left(p_{2}\right)+d\left(p_{2}\right) d(z)\right)\right]\right. \\
& =\sum_{z \varepsilon V\left(G_{2}\right) p_{1} p_{2} E E\left(R_{k}\left(G_{1}\right)\right) p_{1_{1} \varepsilon V}} \sum_{\left(G_{1}\right), p_{2} \varepsilon V\left(R_{k}\left(G_{1}\right)\right)-V\left(G_{1}\right)}\left[2 d\left(p_{1}\right)\left[1+d(z)+d(y)+d^{2}(z)+2 d(z)+2 d^{2}(y)\right]\right. \\
& =\left[M_{2}\left(R_{1}\left(G_{1}\right)\right)-4 M_{2}\left(G_{1}\right)\left[n_{G_{2}}+4 e_{G_{2}}+M_{1}\left(G_{2}\right)+8 e_{G_{1}} e_{G_{2}}+4 e_{G_{1}} M_{1}\left(G_{2}\right),\right.\right. \\
& \sum_{2}^{\prime \prime \prime}=\sum_{z \varepsilon V\left(G_{2}\right)} \sum_{p_{1} p_{2} \varepsilon E\left(R_{k}\left(G_{1}\right)\right) p_{1}, p_{2} \varepsilon V\left(R_{k}\left(G_{1}\right)\right)-V\left(G_{1}\right)}\left[d\left(p_{1}, z\right) d\left(p_{2}, z\right)\right] \\
& =\sum_{z \varepsilon V\left(G_{2}\right)} \sum_{p_{1} p_{2} E E\left(R_{k}\left(G_{1}\right)\right) p_{1} p_{2} \varepsilon V\left(R_{k}\left(G_{1}\right)\right)-V\left(G_{1}\right)}\left[\left(d\left(p_{1}\right)+d\left(p_{1}\right) d(z)\right)\left(d\left(p_{2}\right)+d\left(p_{2}\right) d(z)\right)\right] \\
& =\sum_{z \varepsilon V\left(G_{2}\right) p_{1} \varepsilon V\left(G_{1}\right), p_{2} \varepsilon V\left(R_{k}\left(G_{1}\right)\right)-V\left(G_{1}\right)}\left[4+8 d(y)+4 d^{2}(z)\right]=4(k-1) e_{G_{1}}\left[n_{G_{2}}+4 e_{G_{2}}+M_{1}\left(G_{2}\right)\right] \text {, }
\end{aligned}
$$




$$
\begin{aligned}
& \sum 3=\sum_{z_{1} z_{2} \varepsilon V\left(G_{2}\right)} \sum_{p_{1} p_{2} \varepsilon E\left(R_{k}\left(G_{1}\right)\right) p_{1}, p_{2} \varepsilon V\left(G_{1}\right)}\left[d\left(p_{1}, z_{1}\right) d\left(p_{2}, z_{2}\right)\right] \\
& +\sum_{z_{1} z_{2} \varepsilon V\left(G_{2}\right)} \sum_{p_{1} p_{2} \varepsilon E\left(R_{k}\left(G_{1}\right)\right) p_{1} \varepsilon V\left(G_{1}\right), p_{2} \varepsilon V\left(R_{k}\left(G_{1}\right)\right)-V\left(G_{1}\right)}\left[d\left(p_{1}, z_{1}\right) d\left(p_{2}, z_{2}\right)\right] \\
& +\sum_{z_{1} z_{2} \varepsilon V\left(G_{2}\right)} \sum_{p_{1} p_{2} \varepsilon E\left(R_{k}\left(G_{1}\right)\right) p_{1}, p_{2} \varepsilon V}\left[d\left(p_{k}\left(p_{1}, z_{1}\right)\right)-V\left(G_{1}\right) d\left(p_{2}, z_{2}\right)\right]=\sum 3^{\prime}+\sum 3^{\prime \prime}+\sum 3^{\prime \prime \prime} \\
& \sum 3^{\prime}=\sum_{z_{1} z_{2} \varepsilon V\left(G_{2}\right)} \sum_{p_{1} p_{2} \varepsilon E\left(R_{k}\left(G_{1}\right)\right) p_{1}, p_{2} \varepsilon V\left(G_{1}\right)}\left[d\left(p_{1}, z_{1}\right) d\left(p_{2}, z_{2}\right)\right] \\
& =\sum_{z_{1} z_{2} \varepsilon V\left(G_{2}\right)} \sum_{p_{1} p_{2} \varepsilon E\left(R_{k}\left(G_{1}\right)\right) p_{1}, p_{2} \varepsilon V\left(G_{1}\right)}\left[4 d\left(p_{1}\right) d\left(p_{2}\right)+2\left[d\left(p_{1}\right) d\left(z_{2}\right)+d\left(p_{2}\right) d\left(z_{1}\right)\right]+d\left(z_{1}\right) d\left(z_{2}\right)\right. \\
& \left.+4 d\left(p_{1}\right) d\left(p_{2}\right) d\left(z_{1}\right) d\left(z_{2}\right)+4 d\left(p_{1}\right) d\left(p_{2}\right)\left[d\left(z_{1}\right)+d\left(z_{2}\right)\right]+2\left[d\left(p_{1}\right)+d\left(p_{2}\right)\right] d\left(z_{1}\right) d\left(z_{2}\right)\right] \\
& =8 M_{2}\left(G_{1}\right) e_{G_{2}}+2 M_{1}\left(G_{1}\right) M_{1}\left(G_{2}\right)+2 M_{2}\left(G_{2}\right) e_{G_{1}}+8 M_{2}\left(G_{1}\right)\left[M_{2}\left(G_{2}\right)+M_{1}\left(G_{2}\right)\right]+4 M_{1}\left(G_{1}\right) M_{2}\left(G_{2}\right) \text {, } \\
& \sum 3^{\prime \prime}=\sum_{z_{1} z_{2} \varepsilon V\left(G_{2}\right)} \sum_{p_{1} p_{2} \varepsilon E\left(R_{k}\left(G_{1}\right)\right) p_{1} \varepsilon V\left(G_{1}\right), p_{2} \varepsilon V}\left[d\left(p_{k}, z_{1}\right) d\left(p_{2}, z_{2}\right)\right] \\
& =\sum_{z_{1} z_{2} \varepsilon V\left(G_{2}\right)} \sum_{p_{1} p_{2} \varepsilon E\left(R_{k}\left(G_{1}\right)\right) p_{1} \varepsilon V\left(G_{1}\right), p_{2} \varepsilon V\left(R_{k}\left(G_{1}\right)\right)-V\left(G_{1}\right)}\left[\left(d\left(p_{1}\right)+d\left(z_{1}\right)+d\left(p_{1}\right) d\left(z_{1}\right)\right)\left(d\left(p_{2}\right)+d\left(p_{2}\right) d\left(z_{2}\right)\right)\right] \\
& =\sum_{z_{1} z_{2} \varepsilon V\left(G_{2}\right)} \sum_{p_{1} p_{2} \varepsilon E\left(R_{k}\left(G_{1}\right)\right) p_{1} \varepsilon V\left(G_{1}\right), p_{2} \varepsilon V\left(R_{k}\left(G_{1}\right)\right)-V\left(G_{1}\right)}\left[4 d ( p _ { 1 } ) \left[1+d\left(z_{2}\right)+d\left(z_{1}\right)\right.\right. \\
& \left.\left.+d\left(z_{1}\right) d\left(z_{2}\right)\right]+2\left[d\left(z_{1}\right)+d\left(z_{1}\right) d\left(z_{2}\right)\right]\right] \\
& =\left[M_{2}\left(R_{1}\left(G_{1}\right)\right)-4 M_{2}\left(G_{1}\right)\right]\left[2 e_{G_{2}}+2 M_{1}\left(G_{2}\right)+2 M_{2}\left(G_{2}\right)\right]+4 e_{G_{1}}\left[M_{1}\left(G_{2}\right)+M_{2}\left(G_{2}\right)\right], \\
& \sum_{3}^{\prime \prime \prime}=\sum_{z_{1} z_{2} \varepsilon V\left(G_{2}\right)} \sum_{p_{1} p_{2} \varepsilon E\left(R_{k}(G 1)\right) p_{1}, p_{2} \varepsilon V}\left[d\left(p_{k}, z_{1}\right) d\left(p_{2}, z_{2}\right)\right]
\end{aligned}
$$

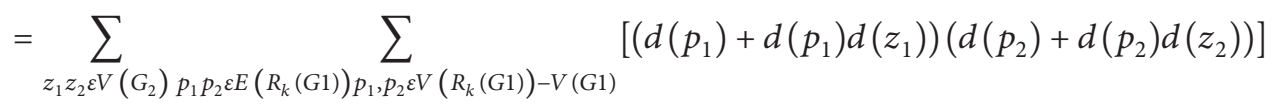

$$
\begin{aligned}
& =\sum_{z_{1} z_{2} \varepsilon V\left(G_{2}\right)} \sum_{p_{1} p_{2} \varepsilon E\left(R_{k}\left(G_{1}\right)\right) p_{1} \varepsilon V\left(G_{1}\right), p_{2} \varepsilon V\left(R_{k}\left(G_{1}\right)\right)-V\left(G_{1}\right)}\left[d\left(p_{1}\right) d\left(p_{2}\right)\left[1+\left(d\left(y_{2}\right)+d\left(z_{1}\right)\right)+d\left(z_{1}\right) d\left(z_{2}\right)\right]\right] \\
& =8(k-1) e_{G_{1}}\left[e_{G_{2}}+M_{1}\left(G_{2}\right)+M_{2}\left(G_{2}\right)\right] .
\end{aligned}
$$

Consequently, we have

$$
\begin{aligned}
M_{2}\left(G_{1} \unrhd_{R_{k}} G_{2}\right)= & M_{1}\left(G_{1}\right)\left[8 e_{G_{2}}+12 M_{1}\left(G_{2}\right)+4 M_{2}\left(G_{2}\right)\right]+4 M_{2}\left(G_{1}\right)\left[n_{G_{2}}+6 e_{G_{2}}+3 M_{1}\left(G_{2}\right)\right] \\
& +\left[M_{2}\left(R_{1}\left(G_{1}\right)\right)-4 M_{2} G_{1}+4(k-1) e_{G_{1}}\right]\left[6 e_{G_{2}}+3 M_{1}\left(G_{2}\right)+2 M_{2}\left(G_{2}\right)+n_{G_{2}}\right] \\
& +M_{2}\left(G_{2}\right)\left[n_{G_{1}}+2 e_{G_{1}}\right]+4 e_{G_{1}}\left[3 M_{1}\left(G_{2}\right)+3 M_{2}\left(G_{2}\right)+4 e_{G_{2}}\right]+e_{G_{1}} M_{1}\left(G_{2}\right) .
\end{aligned}
$$


Theorem 3. Let $G_{1}$ and $G_{2}$ be two connected graphs such that $\left|V\left(G_{1}\right)\right|,\left|V\left(G_{2}\right)\right| \geq 4$. For $k \geq 1$,

(a) $M_{1}\left(G_{1} \otimes_{Q_{k}} G_{2}\right)=k\left[M_{3}\left(G_{1}\right)+2 M_{2}\left(G_{1}\right)-2 M_{1}\left(G_{1}\right)\right]\left[n_{G_{2}}+4 e_{G_{2}}+M_{1}\left(G_{2}\right)\right]+8 e_{G_{1}} e_{G_{2}}$

$$
+M_{1}\left(G_{1}\right)[2(k-1)+3]\left[n_{G_{2}}+4 e_{G_{2}}+M_{1}\left(G_{2}\right)\right]+M_{1}\left(G_{2}\right)\left[n_{G_{1}}+4 e_{G_{1}}\right],
$$

(b) $M_{2}\left(G_{1} \otimes_{Q_{k}} G_{2}\right)=k\left[n_{G_{2}}+6 e_{G_{2}}+3 M_{1}\left(G_{2}\right)+2 M_{2}\left(G_{2}\right)\right]\left[\frac{1}{2} \sum_{v \varepsilon V\left(G_{1}\right)}\left(d_{G_{1}}^{4}(v)-d_{G_{1}}^{3}(v)\right)\right]$

$$
\begin{aligned}
& +\left[\sum_{u v \varepsilon V\left(G_{1}\right)} r d_{G_{1}}(u) d_{G_{1}}(v)+\sum_{v \varepsilon V\left(G_{1}\right)} d_{G_{1}}^{2}(v) \sum_{u \varepsilon V\left(G_{1}\right)} d_{G_{1}}(u)-2 M_{2}\left(G_{1}\right)\right] \\
& +M_{2}\left(G_{2}\right)\left[4 e_{G_{1}}+n_{G_{1}}\right]+2 e_{G_{1}} M_{1}\left(G_{2}\right)+M_{1}\left(G_{1}\right)\left[5 e_{2}+5 M_{1}\left(G_{2}\right)+5 M_{2}\left(G_{2}\right)\right] \\
& +k\left[M_{3}\left(G_{1}\right)+2 M_{2}\left(G_{1}\right)\right]\left[6 e_{G_{2}}+3 M_{1}\left(G_{2}\right)+2 M_{2}\left(G_{2}\right)+n_{G_{2}}\right] .
\end{aligned}
$$

Proof. Let $d(p, z)=d_{G_{1} \otimes_{Q_{G}} G_{2}}(p, z)$ be the degree of a vertex $(p, z)$ in the graph $G_{1} \otimes_{Q_{k}} G_{2}$.

$$
\begin{aligned}
M_{1}\left(G_{1} \otimes_{Q_{k}} G_{2}\right)= & \sum_{(p, z) \varepsilon V\left(G_{1} \otimes_{Q_{k}} G_{2}\right)} d^{2}(p, z)=\sum_{\left(p_{1}, z_{1}\right)\left(p_{2}, z_{2}\right) \varepsilon E\left(G_{1} \otimes_{Q_{k}} G_{2}\right)}\left[d\left(p_{1}, z_{1}\right)+d\left(p_{2}, z_{2}\right)\right] \\
= & \sum_{p \varepsilon V\left(G_{1}\right)} \sum_{z_{1} z_{2} \varepsilon E\left(G_{2}\right)}\left[d\left(p, z_{1}\right)+d\left(p, z_{2}\right)\right]+\sum_{z \varepsilon V\left(G_{2}\right)} \sum_{p_{1} p_{2} \varepsilon E\left(Q_{k}\left(G_{1}\right)\right)}\left[d\left(p_{1}, z\right)+d\left(p_{2}, z\right)\right] \\
& +\sum_{p_{1} p_{2} \varepsilon E\left(Q_{k}\left(G_{1}\right)\right)} \sum_{z_{1} z_{2} \varepsilon V\left(G_{2}\right)}\left[d\left(p_{1}, z_{1}\right)+d\left(p_{2}, z_{2}\right)\right]=\sum 1+\sum 2+\sum 3, \\
\sum 1= & \sum_{p \varepsilon V\left(G_{1}\right)} \sum_{z_{1} z_{2} \varepsilon E\left(G_{2}\right)}\left[d\left(p, z_{1}\right)+d\left(p, z_{2}\right)\right] \\
= & \sum_{p \varepsilon V\left(G_{1}\right)} \sum_{z_{1} z_{2} \varepsilon E\left(G_{2}\right)}\left[d(p)+d\left(z_{1}\right)+d(p) d\left(z_{1}\right)+d(p)+d\left(z_{2}\right)+d(p) d\left(z_{2}\right)\right] \\
= & \sum_{p \varepsilon V(G)} \sum_{z_{1} z_{2} \varepsilon E\left(G_{2}\right)}\left[2 d(p)+d\left(z_{1}\right)+d\left(z_{2}\right)+d(p)\left[d\left(z_{1}\right)+d\left(z_{2}\right)\right]\right] \\
= & 4 e_{G_{1}} e_{G_{2}}+M_{1}\left(G_{2}\right) n_{G_{1}}+2 M_{1}\left(G_{2}\right) e_{G_{1}}, \\
\sum= & \sum_{z \varepsilon V\left(G_{2}\right)} \sum_{p_{1} p_{2} \varepsilon E\left(Q_{k}\left(G_{1}\right)\right)}\left[d\left(p_{1}, z\right)+d\left(p_{2}, z\right)\right] \\
= & \sum_{z \varepsilon V\left(G_{2}\right)} \sum_{p_{1} p_{2} \varepsilon E\left(Q_{k}\left(G_{1}\right)\right) p_{1} \varepsilon V\left(G_{1}\right), p_{2} \varepsilon V\left(Q_{k}\left(G_{1}\right)-G_{1}\right)}\left[d\left(p_{1}, z\right)+d\left(p_{2}, z\right)\right] \\
& +\sum_{z \varepsilon V\left(G_{2}\right)} \sum_{p_{1} p_{2} \varepsilon E\left(Q_{k}\left(G_{1}\right)\right) p_{1}, p_{2} \varepsilon V\left(Q_{k}\left(G_{1}\right)-G_{1}\right)}\left[d\left(p_{1}, y\right)+d\left(p_{2}, z\right)\right]=\sum 2^{\prime}+\sum 2^{\prime \prime},
\end{aligned}
$$




$$
\begin{aligned}
& \sum 2^{\prime}=\sum_{z \varepsilon V\left(G_{2}\right)} \sum_{p_{1} p_{2} \varepsilon E\left(Q_{k}\left(G_{1}\right)\right) p_{1} \varepsilon V\left(G_{1}\right), p_{2} \varepsilon V}\left[d\left(Q_{k}\left(G_{1}\right)\right)-V\left(G_{1}\right)+d\left(p_{2}, z\right)\right] \\
& =\sum_{z \varepsilon V\left(G_{2}\right)} \sum_{p_{1} p_{2} \varepsilon E\left(Q_{k}\left(G_{1}\right)\right) p_{1} \varepsilon V\left(G_{1}\right), p_{2} \varepsilon V\left(Q_{k}\left(G_{1}\right)\right)-V\left(G_{1}\right)}\left[d\left(p_{1}\right)+d(z)+d\left(p_{1}\right) d(y)+d\left(p_{2}\right)+d\left(p_{2}\right) d(z)\right] \\
& =M_{1}\left(G_{1}\right) n_{G_{2}}+4 e_{G_{1}} e_{G_{2}}+6 M_{1}\left(G_{1}\right) e_{G_{2}}+2 n_{G_{2}} M_{1}\left(G_{1}\right) \text {, } \\
& \sum 2^{\prime \prime}=\sum_{z \varepsilon V\left(G_{2}\right)} \sum_{p_{1} p_{2} \varepsilon E\left(Q_{k}\left(G_{1}\right)\right)}\left[d\left(p_{1}, z\right) d\left(p_{2}, z\right)\right] \text {. } \\
& p_{1} p_{2} \varepsilon V\left(Q_{k}\left(G_{1}\right)\right)-V\left(G_{1}\right)
\end{aligned}
$$

Now, we split this sum into two parts for the vertices, $p_{1}$ and $p_{2}$, where $p_{1} p_{2} \varepsilon V\left(Q_{k}\left(G_{1}\right)\right)-V\left(G_{1}\right)$. Assume that $\sum 2^{\prime \prime}=\sum 2^{\prime \prime} a+\sum 2^{\prime \prime} b$, where $\sum 2^{\prime \prime} a$ cover the edges of
$Q_{k}\left(G_{1}\right)$ which are in the same edges of $G_{1}$ and $\sum 2^{\prime \prime} b$ of $Q_{k}\left(G_{1}\right)$ in two different adjacent edges of $G_{1}$.

$$
\begin{aligned}
& \sum 2^{\prime \prime} a=\sum_{z \varepsilon V\left(G_{2}\right)} \sum_{p_{1} p_{2} \varepsilon E} \sum_{\left(Q_{k}\left(G_{1}\right)\right) p_{1}, p_{2} \varepsilon V}\left[d\left(p_{1}, z\right)+d\left(p_{2}, z\right)\right] \\
& =\sum_{z \varepsilon V\left(G_{2}\right)} \sum_{p_{1} p_{2} \varepsilon E\left(Q_{k}\left(G_{1}\right)\right) p_{1}, p_{2} \varepsilon V\left(Q_{k}\left(G_{1}\right)\right)-V\left(G_{1}\right)}\left[d\left(p_{1}\right)+d\left(p_{1}\right) d(z)+d\left(p_{2}\right)+d\left(p_{2}\right) d(z)\right] \\
& =2(k-1) n_{G_{2}} M_{1}\left(G_{1}\right)+4(k-1) e_{G_{2}} M_{1}\left(G_{1}\right), \\
& \sum 2^{\prime \prime} b=\sum_{z \varepsilon V\left(G_{2}\right)} \sum_{p_{1} p_{2} \varepsilon E\left(Q_{k}\left(G_{1}\right)\right) p_{1}, p_{2} \varepsilon V}\left[d\left(p_{1}, y\right)+d\left(p_{2}, z\right)\right] \\
& =\sum_{z \varepsilon V\left(G_{2}\right)} \sum_{p_{1} p_{2} \varepsilon E\left(Q_{k}\left(G_{1}\right)\right)} \sum_{p_{1}, p_{2} \varepsilon V\left(Q_{k}\left(G_{1}\right)\right)-V\left(G_{1}\right)}\left[d\left(p_{1}\right)+d\left(p_{1}\right) d(z)+d\left(p_{2}\right)+d\left(p_{2}\right) d(z)\right]
\end{aligned}
$$

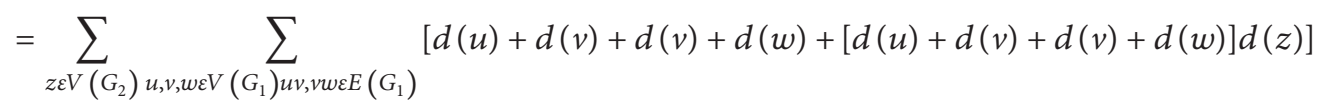

$$
\begin{aligned}
& =k\left[M_{3}\left(G_{1}\right)+2 M_{2}\left(G_{1}\right)-2 M_{1}\left(G_{1}\right)\right]\left[n_{G_{2}}+2 e_{G_{2}}\right], \\
& \sum 3=\sum_{p_{1} p_{2} \varepsilon E\left(Q_{k}\left(G_{1}\right)\right)} \sum_{z_{1} z_{2} \varepsilon V\left(G_{2}\right)}\left[d\left(p_{1}, z_{1}\right)+d\left(p_{2}, z_{2}\right)\right] \\
& =\sum_{z_{1} z_{2} \varepsilon V\left(G_{2}\right)} \sum_{p_{1} p_{2} \varepsilon E\left(Q_{k}\left(G_{1}\right)\right) p_{1} \varepsilon V\left(G_{1}\right), p_{2} \varepsilon V\left(Q_{k}\left(G_{1}\right)\right)-V\left(G_{1}\right)}\left[d\left(p_{1}, z_{1}\right)+d\left(p_{2}, z_{2}\right)\right] \\
& +\sum_{z_{1} z_{2} \varepsilon V\left(G_{2}\right)} \sum_{p_{1} p_{2} \varepsilon E\left(Q_{k}\left(G_{1}\right)\right)} \sum_{p_{1}, p_{2} \varepsilon V}\left[d\left(Q_{k}\left(p_{1}\right)\right)-V\left(z_{1}\right)+d\left(p_{2}, z_{2}\right)\right] \\
& =\sum 3^{\prime}+\sum 3^{\prime \prime} \\
& \sum 3^{\prime}=\sum_{z_{1} z_{2} \varepsilon V\left(G_{2}\right)} \sum_{p_{1} p_{2} \varepsilon E\left(Q_{k}\left(G_{1}\right)\right) p_{1}, p_{2} \varepsilon V\left(G_{1}\right)}\left[d\left(p_{1}, z_{1}\right)+d\left(p_{2}, z_{2}\right)\right] \\
& =\sum_{z_{1} z_{2} \varepsilon V\left(G_{2}\right)} \sum_{p_{1} p_{2} \varepsilon E\left(Q_{k}\left(G_{1}\right)\right) p_{1}, p_{2} \varepsilon V\left(G_{1}\right)}\left[d\left(p_{1}\right)+d\left(z_{1}\right)+d\left(p_{1}\right) d\left(z_{1}\right)+d\left(p_{2}\right)+d\left(z_{2}\right)+d\left(p_{2} z_{2}\right)\right] \\
& =6 e_{G_{2}} M_{1}\left(G_{1}\right)+2 M_{1}\left(G_{2}\right) e_{G_{1}}+3 M_{1}\left(G_{2}\right) M_{1}\left(G_{1}\right) \text {. }
\end{aligned}
$$


Now, we split this sum into two parts for the vertices, $p_{1}$ and $p_{2}$, where $p_{1} p_{2} \in V\left(Q_{k}\left(\Gamma_{1}\right)\right)-V\left(\Gamma_{1}\right)$. Assume that $\sum 3^{\prime \prime}=\sum 3^{\prime \prime} a+\sum 3^{\prime \prime} b$, where $\sum 3^{\prime \prime} a$ cover the edges of
$Q_{k}\left(G_{1}\right)$ which are in the same edges of $G_{1}$ and $\sum 3^{\prime \prime} b$ of $Q_{k}\left(G_{1}\right)$ in two different adjacent edges of $G_{1}$.

$$
\begin{aligned}
\sum 3^{\prime \prime} a & =\sum_{z_{1} z_{2} \varepsilon V\left(G_{2}\right)} \sum_{p_{1} p_{2} \varepsilon E\left(Q_{k}\left(G_{1}\right)\right) p_{1} \varepsilon V\left(G_{1}\right), p_{2} \varepsilon V\left(Q_{k}\left(G_{1}\right)\right)-V\left(G_{1}\right)} \sum_{z_{1} z_{2} \varepsilon V\left(G_{2}\right)} \sum_{p_{1} p_{2} \varepsilon E\left(Q_{k}\left(G_{1}\right)\right) p_{1} \varepsilon V\left(G_{1}\right), p_{2} \varepsilon V\left(Q_{k}\left(G_{1}\right)\right)-V\left(G_{1}\right)}\left[d\left(p_{1}\right)+d\left(p_{1}\right) d\left(z_{1}\right)+d\left(p_{2}\right)+d\left(p_{2}\right) d\left(z_{2}\right)\right] \\
= & 4(k-1) e_{G_{2}} M_{1}\left(G_{1}\right)+2(k-1) M_{1}\left(G_{2}\right) M_{1}\left(G_{1}\right) \\
\sum 3^{\prime \prime} b= & \sum_{z_{1} z_{2} \varepsilon V\left(G_{2}\right) p_{1} p_{2} \varepsilon E\left(Q_{k}\left(G_{1}\right)\right) p_{1}, p_{2} \varepsilon V\left(Q_{k}\left(G_{1}\right)\right)-V\left(G_{1}\right)}\left[d\left(p_{1}, z_{1}\right)+d\left(p_{2}, z_{2}\right)\right] \\
& =\sum_{z_{1} z_{2} \varepsilon V\left(G_{2}\right) p_{1} p_{2} \varepsilon E\left(Q_{k}\left(G_{1}\right)\right) p_{1}, p_{2} \varepsilon V\left(Q_{k}\left(G_{1}\right)\right)-V\left(G_{1}\right)}\left[d\left(p_{1}\right)+d\left(p_{2}\right)+d\left(p_{1}\right) d\left(z_{1}\right)+d\left(p_{2}\right) d\left(z_{2}\right)\right] \\
& =k\left[M_{3}\left(G_{1}\right)+2 M_{2}\left(G_{1}\right)-2 M_{1} G_{1}\right]\left[2 e_{G_{2}}+M_{1}\left(G_{2}\right)\right] .
\end{aligned}
$$

Consequently, we have

$$
\begin{aligned}
M_{1}\left(G_{1} \otimes_{Q_{k}} G_{2}\right)= & k\left[M_{3}\left(G_{1}\right)+2 M_{2}\left(G_{1}\right)-2 M_{1}\left(G_{1}\right)\right]\left[n_{G_{2}}+4 e_{G_{2}}+M_{1}\left(G_{2}\right)\right]+8 e_{G_{1}} e_{G_{2}} \\
& +M_{1}\left(G_{1}\right)[2(k-1)+3]\left[n_{G_{2}}+4 e_{G_{2}}+M_{1}\left(G_{2}\right)\right]+M_{1}\left(G_{2}\right)\left[n_{G_{1}}+4 e_{G_{1}}\right] .
\end{aligned}
$$

Next,

$$
\text { (b) } \begin{aligned}
M_{2}\left(G_{1} \otimes_{Q_{k}} G_{2}\right)= & \sum_{(p, z) \varepsilon V\left(G_{1} \otimes_{Q_{k}} G_{2}\right)} d^{2}(p, z)=\sum_{\left(p_{1}, 1\right)} \sum_{\left(p_{2}, z_{2}\right) \varepsilon E\left(G_{1} \otimes_{Q_{k}} G_{2}\right)}\left[d\left(p_{1}, z_{1}\right) d\left(p_{2}, z_{2}\right)\right] \\
= & \sum_{p \varepsilon V\left(G_{1}\right)} \sum_{z_{1} z_{2} \varepsilon E\left(G_{2}\right)}\left[d\left(p, z_{1}\right) d\left(p, z_{2}\right)\right]+\sum_{z \varepsilon V\left(G_{2}\right)} \sum_{p_{1} p_{2} \varepsilon E\left(Q_{k}\left(G_{1}\right)\right)}\left[d\left(p_{1}, z\right) d\left(p_{2}, z\right)\right] \\
& +\sum_{p_{1} p_{2} \varepsilon E\left(Q_{k}\left(G_{1}\right)\right)} \sum_{z_{1} z_{2} \varepsilon V\left(G_{2}\right)}\left[d\left(p_{1}, z_{1}\right) d\left(p_{2}, z_{2}\right)\right]=\sum 1+\sum 2+\sum 3 .
\end{aligned}
$$


Consider

$$
\begin{aligned}
& \sum 1=\sum_{p \varepsilon V\left(G_{1}\right)} \sum_{z_{1} z_{2} \varepsilon E\left(G_{2}\right)}\left[d\left(p, z_{1}\right) d\left(p, z_{2}\right)\right]=\sum_{p \varepsilon V\left(G_{1}\right)} \sum_{z_{1} z_{2} \varepsilon E\left(G_{2}\right)}\left[d(p)+d\left(z_{1}\right)\right]\left[d(p)+d\left(z_{2}\right)\right] \\
& =M_{1}\left(G_{1}\right) e_{G_{2}}+M_{1}\left(G_{1}\right)\left[M_{1}\left(G_{2}\right)+M_{2}\left(G_{2}\right)\right]+2 e_{G_{1}}\left[M_{1}\left(G_{2}\right)+2 M_{2}\left(G_{2}\right)\right]+M_{2}\left(G_{2}\right) n_{G_{1}}, \\
& \sum 2=\sum_{z \mathcal{E} V\left(G_{2}\right)} \sum_{p_{1} p_{2} \varepsilon E\left(Q_{k}\left(G_{1}\right)\right)}\left[d\left(p_{1}, z\right) d\left(p_{2}, z\right)\right]=\sum_{z \mathcal{E} V\left(G_{2}\right)} \sum_{\substack{p_{1} p_{2} \varepsilon E\left(Q_{k}\left(G_{1}\right)\right) \\
p_{1} \varepsilon V\left(G_{1}\right) p_{2} \varepsilon V\left(Q_{k}\left(G_{1}\right)\right)-V\left(G_{2}\right)}}\left[d\left(p_{1}, z\right) d\left(p_{2}, z\right)\right]
\end{aligned}
$$

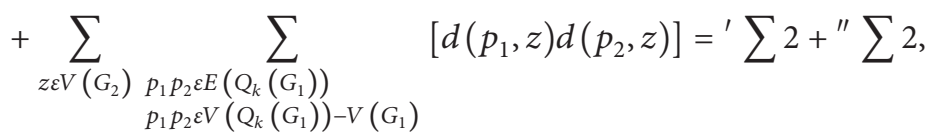

$$
\begin{aligned}
& { }^{\prime} \sum 2=\sum_{z \varepsilon V\left(G_{2}\right)} \sum_{p_{1} p_{2} \varepsilon E\left(Q_{k}\left(G_{1}\right)\right) p_{1} \varepsilon V\left(G_{1}\right) p_{2} \varepsilon V\left(Q_{k}\left(G_{1}\right)\right)-V\left(G_{1}\right)}\left[d\left(p_{1}, z\right) d\left(p_{2}, z\right)\right] \\
& =\sum_{z \varepsilon V\left(G_{2}\right)} \sum_{p_{1} p_{2} \varepsilon E\left(Q_{k}\left(G_{1}\right)\right) p_{1} \varepsilon V\left(G_{1}\right) p_{2} \varepsilon V\left(Q_{k}\left(G_{1}\right)\right)-V\left(G_{1}\right)}\left[d\left(p_{1}\right)+d(z)+d\left(p_{1}\right) d(z)\right]\left[d\left(p_{2}\right)+d(z)+d\left(p_{2}\right) d(z)\right] \\
& =\sum_{z \varepsilon V\left(G_{2}\right)} \sum_{p_{1} p_{2} \varepsilon E\left(Q_{k}\left(G_{1}\right)\right)}\left[d\left(p_{1}\right) d\left(p_{2}\right)\left(1+2 d(z)+d^{2}(z)\right)+d\left(p_{2}\right)\left(d(z)+d^{2}(z)\right)\right] \\
& =\left[M_{3}\left(G_{1}\right)+2 M_{2}\left(G_{1}\right)\right]\left[n_{G_{2}}+4 e_{G_{2}}+M_{1}\left(G_{2}\right)\right]+2 M_{1}\left(G_{1}\right)\left[2 e_{G_{2}}+M_{1}\left(G_{2}\right)\right], \\
& \sum 2^{\prime \prime}=\sum_{z \varepsilon V\left(G_{2}\right)} \sum_{\substack{p_{1} p_{2} \varepsilon E\left(Q_{k}\left(G_{1}\right)\right) \\
p_{1} p_{2} \varepsilon V\left(Q_{k}\left(G_{1}\right)\right)-V\left(G_{1}\right)}}\left[d\left(p_{1}, z\right) d\left(p_{2}, z\right)\right] \text {. }
\end{aligned}
$$

Now, we split this sum into two parts for the vertices, $p_{1}$ $\sum 2^{\prime \prime} a$ which are in the same edges of $G_{1}$ and $\sum 2^{\prime \prime} b$ of and $p_{2}$, where $p_{1} p_{2} \varepsilon V\left(Q_{k}\left(G_{1}\right)\right)-V\left(G_{1}\right)$. Assume that $Q_{k}\left(G_{1}\right)$ in two different adjacent edges of $G_{1}$.

$$
\begin{aligned}
& \sum 2^{\prime \prime} a=\sum_{z \varepsilon V\left(G_{2}\right)} \sum_{\substack{p_{1} p_{2} \varepsilon E\left(Q_{k}\left(G_{1}\right)\right) \\
p_{1} p_{2} \varepsilon V\left(Q_{k}\left(G_{1}\right)\right)-V\left(G_{1}\right)}}\left[\left(d\left(p_{1}\right)+d\left(p_{1}\right) d(z)\right)\left(d\left(p_{2}\right)+d\left(p_{2}\right) d(z)\right)\right] \\
& =\sum_{z \varepsilon V\left(G_{2}\right)} \sum_{\substack{p_{1} p_{2} \varepsilon E\left(Q_{k}\left(G_{1}\right)\right) \\
p_{1} p_{2} \varepsilon V\left(Q_{k}\left(G_{1}\right)\right)-V\left(G_{1}\right)}}\left[d\left(p_{1}\right) d\left(p_{2}\right)+2 d\left(p_{1}\right) d\left(p_{2}\right) d(z)+d\left(p_{1}\right) d\left(p_{2}\right) d^{2}(z)\right] \\
& =\sum_{z \varepsilon V\left(G_{2}\right)} \sum_{\substack{p_{1} p_{2} \varepsilon E\left(Q_{k}\left(G_{1}\right)\right) \\
p_{1} p_{2} \varepsilon V\left(Q_{k}\left(G_{1}\right)\right)-V\left(G_{1}\right)}}\left[d\left(p_{1}\right) d\left(p_{2}\right)\right]\left[1+2 d(z)+d^{2}(z)\right] \\
& \sum 2^{\prime \prime} b=\sum_{z \varepsilon V\left(G_{2}\right)} \sum_{\substack{p_{1} p_{2} \varepsilon E\left(Q_{k}\left(G_{1}\right)\right) \\
p_{1} p_{2} \varepsilon V\left(Q_{k}\left(G_{1}\right)\right)-V\left(G_{1}\right)}}^{(k-1)\left[M_{3}\left(G_{1}\right)+2 M_{2}\left(G_{1}\right)\right]\left[n_{G_{2}}+4 e_{G_{2}}+M_{1}\left(G_{2}\right)\right],}, \\
& =k \sum_{z \varepsilon V\left(G_{2}\right)}\left[1+2 d(z)+d^{2}(z)\right] \sum_{\left.\substack{p_{1} p_{2} \varepsilon E\left(Q_{k}\left(G_{1}\right)\right) \\
p_{1} p_{2} \varepsilon V\left(Q_{k}\left(G_{1}\right)\right.}\right)-V\left(G_{1}\right)}\left[d\left(p_{1}\right) d\left(p_{2}\right)\right] \\
& =k\left[n_{G_{2}}+4 e_{G_{2}}+M_{1}\left(G_{2}\right)\right] \sum_{\substack{u v \varepsilon E\left(G_{1}\right) \\
v w \varepsilon E\left(G_{2}\right)}}\left[d_{G_{1}}(u)+d_{G_{1}}(v)\right]\left[d_{G_{1}}(v)+d_{G_{1}}(w)\right],
\end{aligned}
$$


where $p_{1}$ is the added vertex in the edge $u v$ and $p_{2}$ is added vertex in the edges $v w$ of $G_{1}$ :

$$
\left.\begin{array}{rl}
= & (k)\left[n_{G_{2}}+4 e_{G_{2}}+M_{1}\left(G_{2}\right)\right]\left[\frac{1}{2} \sum_{v \varepsilon V\left(G_{1}\right)}\left(d_{G_{1}}^{4}(v)-d_{G_{1}}^{3}(v)\right)+\sum_{u v \varepsilon V\left(G_{1}\right)} r d_{G_{1}}(u) d_{G_{1}}(v)\right] \\
& +\left[\sum_{v \varepsilon V\left(G_{1}\right)} d_{G_{1}}^{2}(v) \sum_{u \varepsilon V\left(G_{1}\right)} d_{G_{1}}(u)-2 M_{2}\left(G_{1}\right)\right], \\
\operatorname{uv\varepsilon E}\left(G_{1}\right)
\end{array}\right]
$$

where $r$ is the number of neighbors which are common vertices of $u$ and $v$ in $\left(\Gamma_{1}\right)$.

$$
\begin{aligned}
& \sum 3=\sum_{z_{1} z_{2} \varepsilon V\left(G_{2}\right)} \sum_{p_{1} p_{2} \varepsilon E\left(Q_{k}\left(G_{1}\right)\right) p_{1} \varepsilon V\left(G_{1}\right), p_{2} \varepsilon V\left(Q_{k}\left(G_{1}\right)\right)-V\left(G_{1}\right)}\left[d\left(p_{1}, z_{1}\right) d\left(p_{2}, z_{2}\right)\right] \\
& +\sum_{y_{1} z_{2} \varepsilon V\left(G_{2}\right)} \sum_{p_{1} p s_{2} \varepsilon E} \sum_{\left(Q_{k}\left(G_{1}\right)\right)}\left[d\left(p_{1}, p_{2} \varepsilon V\left(z_{1}\right) d\left(p_{2}, z_{2}\right)\right]=\sum 3^{\prime}+\sum 3^{\prime \prime},\right. \\
& \sum 3^{\prime}=\sum_{z_{1} z_{2} \varepsilon V\left(G_{2}\right)} \sum_{p_{1} p_{2} \varepsilon E\left(Q_{k}\left(G_{1}\right)\right) p_{1} \varepsilon V\left(G_{1}\right), p_{2} \varepsilon V}\left[d\left(p_{k}\left(G_{1}\right)\right)-V\left(G_{1}\right) d\left(p_{2}, z_{2}\right)\right] \\
& =\sum_{z_{1} z_{2} \varepsilon V\left(G_{2}\right) p_{1} p_{2} \varepsilon E\left(Q_{k}\left(G_{1}\right)\right) p_{1} \varepsilon V\left(G_{1}\right), p_{2} \varepsilon V\left(Q_{k}\left(G_{1}\right)\right)-V\left(G_{1}\right)}\left[\left(d\left(p_{1}\right)+d\left(z_{1}\right)+d\left(p_{1}\right) d\left(z_{1}\right)\right)\left(d\left(p_{2}\right)+d\left(p_{2}\right) d\left(z_{2}\right)\right)\right] \\
& =\left[M_{3}\left(G_{1}\right)+2 M_{2}\left(G_{1}\right)\right]\left[2 e_{G_{2}}+2 M_{1}\left(G_{2}\right)+2 M_{2}\left(G_{2}\right)\right]+2 M_{1}\left(G_{1}\right)\left[2 M_{2}\left(G_{2}\right)+M_{1}\left(G_{2}\right)\right], \\
& \sum 3^{\prime \prime}=\sum_{z_{1} z_{2} \varepsilon V\left(G_{2}\right)} \sum_{p_{1} p_{2} \varepsilon E\left(Q_{k}\left(G_{1}\right)\right)}\left[d\left(p_{1}, z\right) d\left(p_{2}, z\right)\right] . \\
& p_{1} p_{2} \varepsilon V\left(Q_{k}\left(G_{1}\right)\right)-V\left(G_{1}\right)
\end{aligned}
$$

Now, we split this sum into two parts for the vertices, $p_{1}$ and $p_{2}$, where $p_{1} p_{2} \varepsilon V\left(Q_{k}\left(G_{1}\right)\right)-V\left(G_{1}\right)$. Assume that $\sum 3^{\prime \prime} a$ which are in the same edges of $G_{1}$ and $\sum 3^{\prime \prime} b$ of $Q_{k}\left(G_{1}\right)$ in two different adjacent edges of $G_{1}$. 


$$
\begin{aligned}
& \sum 3^{\prime \prime} a=\sum_{z_{1} z_{2} \varepsilon V\left(G_{2}\right)} \sum_{p_{1} p_{2} \varepsilon E\left(Q_{k}(G 1)\right) p_{1}, p_{2} \varepsilon V}\left[d\left(p_{1}, z_{1}\right) d\left(p_{2}, z_{2}\right)\right]
\end{aligned}
$$

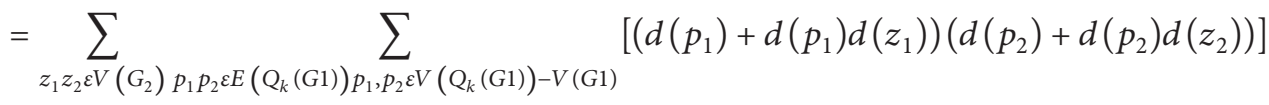

$$
\begin{aligned}
& =\sum_{z_{1} z_{2} \varepsilon V\left(G_{2}\right)}\left[1+d\left(z_{2}\right)+d\left(z_{1}\right)+d\left(z_{1}\right) d\left(z_{2}\right)\right] \sum_{p_{1} p_{2} \varepsilon E\left(Q_{k}\left(G_{1}\right)\right) p_{1} \varepsilon V\left(G_{1}\right), p_{2} \varepsilon V\left(Q_{k}\left(G_{1}\right)\right)-V\left(G_{1}\right)}\left[d\left(p_{1}\right) d\left(p_{2}\right)\right] \\
& =(k-1)\left[2 e_{G_{2}}+2 M_{1}\left(G_{2}\right)+2 M_{2}\left(G_{2}\right)\right]\left[M_{3}\left(G_{1}\right)+2 M_{2}\left(G_{1}\right)\right] \text {, } \\
& \sum 3^{\prime \prime} b=\sum_{z_{1} z_{2} \varepsilon V\left(G_{2}\right)} \sum_{p_{1} p_{2} \varepsilon E} \sum_{\left(Q_{k}(G 1)\right) p_{1}, p_{2} \varepsilon V}\left[d\left(p_{1}, z_{1}\right) d\left(p_{2}, z_{2}\right)\right]
\end{aligned}
$$

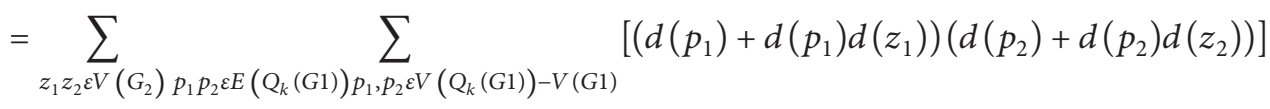

$$
\begin{aligned}
& =\sum_{z_{1} z_{2} \varepsilon V\left(G_{2}\right)}\left[1+d\left(z_{2}\right)+d\left(z_{1}\right)+d\left(z_{1}\right) d\left(z_{2}\right)\right] \sum_{p_{1} p_{2} \varepsilon E\left(Q_{k}\left(G_{1}\right)\right) p_{1} \varepsilon V\left(G_{1}\right), p_{2} \varepsilon V\left(Q_{k}\left(G_{1}\right)\right)-V\left(G_{1}\right)}\left[d\left(p_{1}\right) d\left(p_{2}\right)\right] \\
& =k\left[2 e_{G_{2}}+2 M_{1}\left(G_{2}\right)+2 M_{2}\left(G_{2}\right)\right] \sum_{\substack{u v E E\left(G_{1}\right) \\
v w \varepsilon E\left(G_{2}\right)}}\left[d_{G_{1}}(u)+d_{G_{1}}(v)\right]\left[d_{G_{1}}(v)+d_{G_{1}}(w)\right],
\end{aligned}
$$

where $p_{1}$ is the added vertex in the edge $u v$ and $p_{2}$ is added vertex in the edges $v w$ of $G_{1}$ :

$$
\begin{aligned}
& =(k)\left[2 e_{G_{2}}+2 M_{1}\left(G_{2}\right)+2 M_{2}\left(G_{2}\right)\right]\left[\frac{1}{2} \sum_{v \varepsilon V\left(G_{1}\right)}\left(d_{G_{1}}^{4}(v)-d_{G_{1}}^{3}(v)\right)+\sum_{u v \varepsilon V\left(G_{1}\right)} r d_{G_{1}}(u) d_{G_{1}}(v)\right. \\
& \left.+\sum_{v \varepsilon V\left(G_{1}\right)} d_{G_{1}}^{2}(v) \sum_{u \varepsilon V\left(G_{1}\right)} d_{G_{1}}(u)-2 M_{2}\left(G_{1}\right)\right]
\end{aligned}
$$

where $r$ is the number of neighbors which are common vertices of $u$ and $v$ in $\left(G_{1}\right)$.

Consequently, we have

$$
\begin{aligned}
M_{2}\left(G_{1} \otimes_{Q_{k}} G_{2}\right)= & k\left[n_{G_{2}}+6 e_{G_{2}}+3 M_{1}\left(G_{2}\right)+2 M_{2}\left(G_{2}\right)\right]\left[\frac{1}{2} \sum_{v \varepsilon V\left(G_{1}\right)}\left(d_{G_{1}}^{4}(v)-d_{G_{1}}^{3}(v)\right)\right. \\
& \left.+\sum_{u v \varepsilon V\left(G_{1}\right)} r d_{G_{1}}(u) d_{G_{1}}(v)+\sum_{v \varepsilon V\left(G_{1}\right)} d_{G_{1}}^{2}(v) \sum_{u \varepsilon V\left(G_{1}\right)} d_{G_{1}}(u)-2 M_{2}\left(G_{1}\right)\right] \\
& +M_{2}\left(G_{2}\right)\left[4 e_{G_{1}}+n_{G_{1}}\right]+2 e_{G_{1}} M_{1}\left(G_{2}\right)+M_{1}\left(G_{1}\right)\left[5 e_{2}+5 M_{1}\left(G_{2}\right)+5 M_{2}\left(G_{2}\right)\right] \\
& +k\left[M_{3}\left(G_{1}\right)+2 M_{2}\left(G_{1}\right)\right]\left[6 e_{G_{2}}+3 M_{1}\left(G_{2}\right)+2 M_{2}\left(G_{2}\right)+n_{G_{2}}\right] .
\end{aligned}
$$


Theorem 4. Let $G_{1}$ and $G_{2}$ be two connected graphs such that $\left|V\left(G_{1}\right)\right|,\left|V\left(G_{2}\right)\right| \geq 4$. For $k \geq 1$,

(a) $M_{1}\left(G_{1} \otimes_{T_{k}} G_{2}\right)=M_{1}\left(G_{2}\right)\left[8 e_{G_{1}}+n_{G_{1}}+6 M_{1}\left(G_{1}\right)\right]+2(k-1) M_{1}\left(G_{1}\right)\left[2 e_{G_{2}}+M_{1}\left(G_{2}\right)\right]$

$$
\begin{aligned}
& +k\left[M_{3}\left(G_{1}\right)+2 M_{2}\left(G_{1}\right)-2 M_{1}\left(G_{1}\right)\right]\left[4 e_{G_{2}}+n_{G_{2}}+M_{1}\left(G_{2}\right)\right]+16 e_{G_{1}} e_{G_{2}} \\
& +M_{1}\left(G_{1}\right)\left[2(11+k) e_{G_{2}}+2(k+2) n_{G_{2}}\right]
\end{aligned}
$$

(b) $M_{2}\left(G_{1} \otimes_{T_{k}} G_{2}\right)=k\left[n_{G_{2}}+6 e_{G_{2}}+3 M_{1}\left(G_{2}\right)+2 M_{2}\left(G_{2}\right)\right]\left[\frac{1}{2} \sum_{v \varepsilon V\left(G_{1}\right)}\left(d_{G_{1}}^{4}(v)-d_{G_{1}}^{3}(v)\right)+\sum_{u v \varepsilon V\left(G_{1}\right)} r d_{G_{1}}(u) d_{G_{1}}(v)\right.$

$$
\begin{aligned}
& \left.+\sum_{\substack{v \varepsilon V\left(G_{1}\right) \\
G_{G_{1}}}}^{2}(v) \sum_{u \varepsilon V\left(G_{1}\right)} d_{G_{1}}(u)-2 M_{2}\left(G_{1}\right)\right]+M_{2}\left(G_{1}\right)\left[4 n_{G_{2}}+24 e_{G_{2}}+8 M_{2}\left(G_{2}\right)+12 M_{1}\left(G_{2}\right)\right] \\
& +k\left[M_{3}\left(G_{1}\right)+2 M_{2}\left(G_{1}\right)\right]\left[6 e_{G_{2}}+n_{G_{2}}+3 M_{1}\left(G_{2}\right)+2 M_{2}\left(G_{2}\right)\right]+5 M_{1}\left(G_{2}\right) e_{G_{1}} \\
& +M_{2}\left(G_{2}\right)\left[10 e_{G_{1}}+n_{G_{1}}\right]+M_{1}\left(G_{1}\right)\left[10 e_{G_{2}}+11 M_{1}\left(G_{2}\right)+10 M_{2}\left(G_{2}\right)\right] .
\end{aligned}
$$

\section{Applications and Conclusion}

In this section, we have computed the first and second Zagreb indices of generalized $\Phi$-sum graphs based on strong product as application of Theorem 1 to Theorem 4 for $k=1$ given as follows. We also find the subclass with better Zagreb indices as presented in Tables 1 and 2 and Figure 4

(i) $S_{1}$-sum:

$$
\begin{aligned}
\text { (a) } M_{1}\left(G_{1} \otimes_{S_{1}} G_{2}\right)= & M_{1}\left(S_{1} G_{1}\right)\left[n_{G_{2}}+2 e_{G_{2}}\right]+8 e_{G_{1}} e_{G_{2}}+\left[M_{1}\left(G_{1}\right)+4 e_{G_{1}}\right]\left[M_{1}\left(G_{2}\right)+2 e_{G_{2}}\right] \\
& +M_{1}\left(G_{2}\right)\left[4 e_{G_{1}}+n_{G_{1}}\right] \\
\text { (b) } M_{2}\left(G_{1} \otimes_{S_{1}} G_{2}\right)= & {\left[M_{2}\left(G_{1}\right)+4 e_{G_{1}}\right]\left[5 e_{G_{2}}+3 M_{1}\left(G_{2}\right)+2 M_{2}\left(G_{2}\right)+n_{G_{2}}\right]+14 e_{G_{1}} M_{1}\left(G_{2}\right) } \\
& \left.+M_{2}\left(G_{2}\right)\right]\left[12 e_{G_{1}}+n_{G_{1}}\right]+M_{1}\left(G_{1}\right)\left[e_{G_{2}}+M_{1}\left(G_{2}\right)+M_{2}\left(G_{2}\right)\right]+8 e_{G_{1}} e_{G_{2}} .
\end{aligned}
$$

(ii) $R_{1}$-sum:

$$
\text { (a) } \begin{aligned}
M_{1}\left(G_{1} \unrhd_{R_{1}} G_{2}\right)= & 24 e_{G_{1}} e_{G_{2}}+M_{1}\left(G_{2}\right)\left[n_{G_{1}}+12 e_{G_{1}}+M_{1}\left(G_{1}\right)\left[12 e_{G_{2}}+2 n_{G_{2}}\right]\right. \\
& +\left[M_{1}\left(R_{1}\left(G_{1}\right)\right)-2 M_{1} G_{1}\right]\left[n_{G_{2}}+2 e_{G_{2}}\right] \\
\text { (b) } M_{2}\left(G_{1} \unrhd_{R_{1}} G_{2}\right)= & 2 M_{1}\left(G_{1}\right)\left[4 e_{G_{2}}+6 M_{1}\left(G_{2}\right)+2 M_{2}\left(G_{2}\right)\right]+4 e_{G_{1}}\left[3 M_{1}\left(G_{2}\right)+3 M_{2}\left(G_{2}\right)+4 e_{G_{2}}\right] \\
& +\left[M_{2}\left(R_{1}\left(G_{1}\right)\right)-4 M_{2} G_{1}\right]\left[6 e_{G_{2}}+3 M_{1}\left(G_{2}\right)+2 M_{2}\left(G_{2}\right)+n_{G_{2}}\right]+e_{G_{1}} M_{1}\left(G_{2}\right) \\
& +4 M_{2}\left(G_{1}\right)\left[n_{G_{2}}+6 e_{G_{2}}+3 M_{1}\left(G_{2}\right)\right]+M_{2}\left(G_{2}\right)\left[n_{G_{1}}+2 e_{G_{1}}\right] .
\end{aligned}
$$


TABLE 1: $M_{1}\left(P_{n} \otimes_{\Phi_{1}} P_{m}\right)$ for path graph.

\begin{tabular}{|c|c|c|c|c|}
\hline$[\mathrm{m}, \mathrm{n}]$ & $M_{1}\left(P_{n} \bigotimes_{S_{1}} P_{m}\right)$ & $M_{1}\left(P_{n} \otimes_{R_{1}} P_{m}\right)$ & $M_{1}\left(P_{n} \otimes_{Q_{1}} P_{m}\right)$ & $M_{1}\left(P_{n} \bigotimes_{T_{1}} P_{m}\right)$ \\
\hline$(3,3)$ & 336 & 722 & 472 & 892 \\
\hline$(4,4)$ & 804 & 1776 & 1220 & 2204 \\
\hline$(5,5)$ & 1086 & 3294 & 2296 & 4108 \\
\hline$(6,6)$ & 1854 & 7040 & 3700 & 8368 \\
\hline$(7,7)$ & 2606 & 9878 & 5432 & 11848 \\
\hline
\end{tabular}

TABLE 2: $M_{2}\left(P_{n} \varpi_{\Phi_{1}} P_{m}\right)$ for path graph.

\begin{tabular}{|c|c|c|c|c|}
\hline$[\mathrm{m}, \mathrm{n}]$ & $M_{2}\left(P_{n} \bigotimes_{S_{1}} P_{m}\right)$ & $M_{2}\left(P_{n} \otimes_{R_{1}} P_{m}\right)$ & $M_{2}\left(P_{n} \otimes_{Q_{1}} P_{m}\right)$ & $M_{2}\left(P_{n} \otimes_{T_{1}} P_{m}\right)$ \\
\hline$(3,3)$ & 824 & 2384 & 1535 & 2899 \\
\hline$(4,4)$ & 2262 & 5230 & 5002 & 9700 \\
\hline$(5,5)$ & 4404 & 11636 & 9674 & 17818 \\
\hline$(6,6)$ & 6738 & 17546 & 15818 & 28696 \\
\hline$(7,7)$ & 10740 & 25816 & 23026 & 40942 \\
\hline
\end{tabular}

(iii) $Q_{1}$-sum:

$$
\begin{aligned}
\text { (a) } M_{1}\left(G_{1} \otimes_{Q_{1}} G_{2}\right)= & {\left[M_{3}\left(G_{1}\right)+2 M_{2}\left(G_{1}\right)-2 M_{1}\left(G_{1}\right)\right]\left[n_{G_{2}}+4 e_{G_{2}}+M_{1}\left(G_{2}\right)\right]+8 e_{G_{1}} e_{G_{2}} } \\
& +3 M_{1}\left(G_{1}\right)\left[n_{G_{2}}+4 e_{G_{2}}+M_{1}\left(G_{2}\right)\right]+M_{1}\left(G_{2}\right)\left[n_{G_{1}}+4 e_{G_{1}}\right] \\
\text { (b) } M_{2}\left(G_{1} \otimes_{Q_{1}} G_{2}\right)= & {\left[n_{G_{2}}+6 e_{G_{2}}+3 M_{1}\left(G_{2}\right)+2 M_{2}\left(G_{2}\right)\right]\left[\frac{1}{2} \sum_{v \varepsilon V\left(G_{1}\right)}\left(d_{G_{1}}^{4}(v)-d_{G_{1}}^{3}(v)\right)\right.} \\
& \left.+\sum_{v v \varepsilon\left(G_{1}\right)} r d_{G_{1}}(u) d_{G_{1}}(v)+\sum_{v \varepsilon V\left(G_{1}\right)} d_{G_{1}}^{2}(v) \sum_{u \varepsilon V\left(G_{1}\right)} d_{G_{1}}(u)-2 M_{2}\left(G_{1}\right)\right] \\
& +M_{2}\left(G_{2}\right)\left[4 e_{G_{1}}+n_{G_{1}}\right]+2 e_{G_{1}} M_{1}\left(G_{2}\right)+M_{1}\left(G_{1}\right)\left[5 e_{2}+5 M_{1}\left(G_{2}\right)+5 M_{2}\left(G_{2}\right)\right] \\
& +\left[M_{3}\left(G_{1}\right)+2 M_{2}\left(G_{1}\right)\right]\left[6 e_{G_{2}}+3 M_{1}\left(G_{2}\right)+2 M_{2}\left(G_{2}\right)+n_{G_{2}}\right]
\end{aligned}
$$

(iv) $T_{1}$-sum:

(a) $M_{1}\left(G_{1} \otimes_{T_{1}} G_{2}\right)=M_{1}\left(G_{2}\right)\left[8 e_{G_{1}}+n_{G_{1}}+6 M_{1}\left(G_{1}\right)\right]+M_{1}\left(G_{1}\right)\left[24 e_{G_{2}}+6 n_{G_{2}}\right]$

$$
+\left[M_{3}\left(G_{1}\right)+2 M_{2}\left(G_{1}\right)-2 M_{1}\left(G_{1}\right)\right]\left[4 e_{G_{2}}+n_{G_{2}}+M_{1}\left(G_{2}\right)\right]+16 e_{G_{1}} e_{G_{2}},
$$

(b) $M_{2}\left(G_{1} \otimes_{T_{1}} G_{2}\right)=\left[n_{G_{2}}+6 e_{G_{2}}+3 M_{1}\left(G_{2}\right)+2 M_{2}\left(G_{2}\right)\right]\left[\frac{1}{2} \sum_{v \varepsilon V\left(G_{1}\right)}\left(d_{G_{1}}^{4}(v)-d_{G_{1}}^{3}(v)\right)\right.$

$$
\begin{aligned}
& \left.+\sum_{u v \varepsilon V\left(G_{1}\right)} r d_{G_{1}}(u) d_{G_{1}}(v)+\sum_{v \varepsilon V\left(G_{1}\right)} d_{G_{1}}^{2}(v) \sum_{\substack{u \varepsilon V\left(G_{1}\right) \\
u v \varepsilon E\left(G_{1}\right)}} d_{G_{1}}(u)-2 M_{2}\left(G_{1}\right)\right] \\
& +\left[M_{3}\left(G_{1}\right)+2 M_{2}\left(G_{1}\right)\right]\left[6 e_{G_{2}}+n_{G_{2}}+3 M_{1}\left(G_{2}\right)+2 M_{2}\left(G_{2}\right)\right]+5 M_{1}\left(G_{2}\right) e_{G_{1}} \\
& +M_{2}\left(G_{2}\right)\left[10 e_{G_{1}}+n_{G_{1}}\right]+M_{1}\left(G_{1}\right)\left[10 e_{G_{2}}+11 M_{1}\left(G_{2}\right)+10 M_{2}\left(G_{2}\right)\right] \\
& +M_{2}\left(G_{1}\right)\left[4 n_{G_{2}}+24 e_{G_{2}}+8 M_{2}\left(G_{2}\right)+12 M_{1}\left(G_{2}\right)\right] .
\end{aligned}
$$


Now, we close our discussion with the conclusion that both the Zagreb indices of the generalized T-sum graph are dominant among the Zagreb indices of all the generalized sum graphs as shown in Figure 4. We also conclude that, in the generalized T-sum graph, the number of vertices (atoms) and edges (bonds) between them are more than the other graphs of this family for each integral values of $k$. Thus, the role of Zagreb indices for generalized T-sum graph remains dominant for each integral values of $k$. However, the problem is still open to find different degree- and distancebased TIs for the generalized sum graphs obtained under the various operations of product of graphs.

\section{Data Availability}

The data used to support the findings of this study are included within the article. Additional data can be obtained from the corresponding author upon request.

\section{Conflicts of Interest}

The authors declare no conflicts of interest.

\section{Acknowledgments}

This work was supported by University of Tabuk, Tabuk, Saudi Arabia.

\section{References}

[1] I. Gutman, B. Ruscic, N. Trinajstic, and C. F. Wilcox, "Graph theory and molecular orbitals. XII. Acyclic polyenes," The Journal of Chemical Physics, vol. 62, no. 9, pp. 3399-3405, 1975.

[2] L. B. Kier and L. H. Hall, "Structure-activity studies on hallucinogenic amphetamines using molecular connectivity," Journal of Medicinal Chemistry, vol. 20, no. 12, pp. 1631-1636, 1977.

[3] O. M. Minailiuc and M. V. Diudea, "TI-MTD model. applications in molecular design," $Q S P R=Q S A R$ Studies by Molecular Descriptors, pp. 363-388, 2001.

[4] H. Wiener, "Structural determination of paraffin boiling points," Journal of the American Chemical Society, vol. 69, no. 1, pp. 17-20, 1947.

[5] I. Gutman and N. Trinajsti, "Graph theory and molecular orbitals. Total $\varphi$-electron energy of alternant hydrocarbons," Chemical Physics Letters, vol. 17, no. 4, pp. 535-538, 1972.

[6] I. Gutman and N. Trinajstic, Graph Theory and Molecular Orbitals, vol. 2, pp. 49-93, In New Concept, Berlin, Heidelberg, 1973.

[7] Y.-M. Chu, S. Javed, M. Javaid, and M. Kamran Siddiqui, "On bounds for topological descriptors of $\varphi$-sum graphs," Journal of Taibah University for Science, vol. 14, no. 1, pp. 1288-1301, 2020.

[8] G. Hong, Z. Gu, M. Javaid, H. M. Awais, and M. K. Siddiqui, "Degree-based topological invariants of metal-organic networks," IEEE Access, vol. 8, pp. 68288-68300, 2020.

[9] W. Yan, B.-Y. Yang, and Y.-N. Yeh, "The behavior of Wiener indices and polynomials of graphs under five graph decorations," Applied Mathematics Letters, vol. 20, no. 3, pp. 290-295, 2007.
[10] M. Eliasi and B. Taeri, "Four new sums of graphs and their Wiener indices," Discrete Applied Mathematics, vol. 157, no. 4, pp. 794-803, 2009.

[11] J.-B. Liu, S. Javed, M. Javaid, and K. Shabbir, "Computing first general Zagreb index of operations on graphs," IEEE Access, vol. 7, pp. 47494-47502, 2019.

[12] J. B. Liu, M. Javaid, and H. M. Awais, "Computing Zagreb indices of the subdivision-related generalized operations of graphs," IEEE Access, vol. 7, pp. 105479-105488, 2019.

[13] H. M. Awais, M. Javaid, and M. Jamal, "Forgotten index of generalized F-sum graphs," Journal of Prime Research in Mathematics, vol. 15, pp. 115-128, 2019.

[14] H. M. Awais, M. Javaid, and A. Raheem, "Hyper-Zagreb index of graphs based on generalized subdivision related operations," Punjab University Journal of Mathematics, vol. 52, no. 5, pp. 89-103, 2019.

[15] H. M. Awais, M. Javaid, and A. Akbar, "First general zagreb index of generalized F-sum graphs," Discrete Dynamics in Nature and Society, vol. 2020, Article ID 2954975, 16 pages, 2020.

[16] A. Ali, "Tetracyclic graphs with maximum second Zagreb index: a simple approach," Asian-European Journal of Mathematics, vol. 11, no. 05, p. 1850064, 2018.

[17] H. M. Awais, M. Jamal, and M. Javaid, "Topological properties of metal-organic frameworks," Main Group Metal Chemistry, vol. 43 , no. 1 , pp. $67-76,2020$.

[18] X. Zhang, H. M. Awais, M. Javaid, and M. K. Siddiqui, "Multiplicative zagreb indices of molecular graphs," Journal of Chemistry, pp. 1-19, 2019.

[19] H. Deng, D. Sarala, S. K. Ayyaswamy, and S. Balachandran, "The Zagreb indices of four operations on graphs," Applied Mathematics and Computation, vol. 275, pp. 422-431, 2016. 